\title{
Moduli space actions on the Hochschild co-chains of a Frobenius algebra II: correlators
}

\author{
Ralph M. Kaufmann
}

\begin{abstract}
This is the second of two papers in which we prove that a cell model of the moduli space of curves with marked points and tangent vectors at the marked points acts on the Hochschild co-chains of a Frobenius algebra. We also prove that there is dg-PROP action of a version of Sullivan chord diagrams which acts on the normalized Hochschild co-chains of a Frobenius algebra. These actions lift to operadic correlation functions on the co-cycles. In particular, the PROP action gives an action on the homology of a loop space of a compact simply connected manifold.

In this second part, we discretize the operadic and PROPic structures of the first part. We also introduce the notion of operadic correlation functions and use them in conjunction with operadic maps from the cell level to the discretized objects to define the relevant actions.
\end{abstract}

Mathematics Subject Classification (2000). 55P48; 16E40, 17Axx, 18D50, 32G15, 32G81, $81 \mathrm{~T} 40$.

Keywords. Moduli spaces, operads, Hochschild cohomology, foliations, cell models, string topology, Sullivan chord diagrams, PROPs, conformal field theory, correlation functions.

\section{Introduction}

The subject of this sequence of two papers are actions on the Hochschild complex of an associative or, more restrictively, a Frobenius algebra induced by operadic structures on moduli spaces of curves. Our approach is from the point of view of combinatorial field theory, which relies on a graph description of moduli space that also allows for a natural compactification given by Penner; see [KS2], [Co] for different approaches. One upshot of our treatment is that the role of the Frobenius condition becomes clear, thus allowing us to separate when this additional assumption is needed and when one can get by with just an associative algebra. Actions of a cell model of the open moduli space are expected on the grounds of $D$-brane considerations [KR], [KLi1], [KLi2], ${ }^{1}$ while a subspace of moduli space given by certain graphs is supposed to act by the considerations of string topology [CS], [V2], [CJ], [C], [CG], [Ch], [Me2], [S2],

\footnotetext{
${ }^{1} \mathrm{~A}$ more extensive discussion of these links is given in $\$ 5$.
} 
[S1]. The archetype of these actions was established with the proofs of Deligne's conjecture [Ko4], [T], [MS1], [MS2], [MS3], [V1], [KS1], [MS2], [BF], [K4], and its generalizations to the $A_{\infty}$ case [KS1], [KSch], and to the cyclic case [K2]. The essential role of the topological operad in this "classical" case was clearly assigned to the little discs and the framed little discs operads. In the present setup the first objective is to establish the existence of the topological and cellular operads needed to make the above expectations into provable statements. This task was completed in the first part [K5]. For instance one of the results of loc. cit. is that there is a rational operad structure on the chains of the moduli space $M_{g, n+1}^{1^{n+1}}$ of genus $g$ curves with $n$ punctures and a tangent vector at each of these punctures which induces a chain level operad. This result can be seen as a presentation of a combinatorial version of conformal field theory (CFT) in terms of foliations [KP]. As explained in the first part [K5], in our setup the operad structure for the moduli space on the topological level cannot be expected to be strict, since it is only well defined almost everywhere. This is captured by the notion "rational". We also showed that there are topological and chain level operads/PROPs for the subspaces of Sullivan chord diagrams which are at the heart of string topology like operations. Here for the PROP structure we need a weakening to a "quasi"-PROP, which means that the associativity only needs to hold up to homotopy. An important result of the first part is that these weaker structures nevertheless induce the strict operad/PROP structures on the cell level.

In the present second part, we establish that the cell level structures indeed act on the Hochschild co-chains. Our main tool is the notion of operadic correlation functions, which should be understood as a suitable definition of a dg algebra $(A, d)$ over a cyclic operad. Another way to phrase this is that these correlation functions reflect the fact that OPEs in physics are actually only defined within correlators and only on BRST closed fields.

In particular, using the results of [K5], we prove the following theorem announced in loc. cit.:

Theorem A. The moduli space $M_{g, n+1}^{1^{n+1}}$ of genus $g$ curves with $n$ punctures and a tangent vector at each of these punctures has the structure of a rational cyclic operad. This structure induces a cyclic dg operad structure on a cell model computing the cohomology of $M_{g, n+1}^{1^{n+1}}$.

Furthermore the cell level operad operates on the Hochschild co-chains of a Frobenius algebra. It also yields correlation functions on the tensor algebra of the co-cycles of a differential algebra $(A, d)$ with a cyclically invariant trace $\int: A \rightarrow k$ which satisfies $\int d a=0$ and whose induced pairing on $H=H(A, d)$ turns $H$ into a Frobenius algebra.

As stipulated in [K5], there is also a PROPic version of this action involving (a partial compactification of) a subspace. The corresponding theorem pertaining to string topology type operations is again proved in this second part. 
Theorem B. There is a rational topological quasi-PROP which is homotopic to a $C W$ complex whose cellular chains are isomorphic as a free Abelian group to a certain type of Sullivan chord diagrams. These chains form a dg-PROP and hence induce this structure on the chord diagrams. Furthermore if $H$ is a Frobenius algebra there is a PROPic action on the Hochschild co-chains of $H$ that is a dg action. This dg action of a dg-PROP on the dg algebra of Hochschild co-chains naturally descends to an action of the homology of the CW-complex on the Hochschild cohomology of a Frobenius algebra.

Moreover, for $\left(A, d, \int, H\right)$ as in Theorem $A$, the action on $H$ is induced by correlation functions on the tensor algebra of $A$ that yield operadic correlation functions on the tensor algebra of the co-cycles of $A$ for any $(A, d)$ as above.

Finally, the BV operator, which is given by the action of the sub-PROP equivalent to the framed little discs operad, acts as in [K2]. Thus the BV operator for the action on the Hochschild cohomology of $H$ is identified with Connes operator $B$ under the identification of the Hochschild cohomology of a Frobenius algebra with its cyclic cohomology of $H$.

The application to the homology of the loop space of a simply connected manifold then comes as an immediate consequence using Jones' $[\mathrm{J}]$ cyclic description of the free loop space.

Corollary. When taking field coefficients, the above action gives a dg action of a dgPROP of Sullivan chord diagrams on the $E^{1}$-term of a spectral sequence converging to $H_{*}(L M)$, the homology of the loop space of a simply connected compact manifold, and hence induces operations on the homology of the loop space.

If we are not dealing with an algebra $A$ but only with a $\operatorname{dg}$ vector space $(V, d)$ that has a pairing $\langle$,$\rangle which is symmetric and satisfies for all v, w \in V:\langle d v, w\rangle+$ $\langle v, d w\rangle=0$, such that $H=H(V, d)$ is finite and the induced pairing on it is non-degenerate, there is a still an action.

Theorem C. The operads and PROPs above also act on the tensor algebra TV of a triple $(V, d,\langle\rangle$,$) as specified above and yield operadic correlation functions for the$ co-cycles of $T V$.

This action is different from the algebra case of Theorems A and B, making the result interesting in its own right. It seems that there are two basic strategies to obtain correlators from graphs on surfaces, a "multiplicative" action for an algebra and an "additive" action for a vector space. One added feature of this action "descends" to an action of the stabilized arc operad, which forms a spectrum [K6].

As mentioned above, the proof of these facts consists mainly of two steps: first defining the respective topological objects and then defining their actions. The first 
step is contained in [K5] and the second one is the content of this article, in which we define the actions of the various objects. The definition of the action itself again breaks down into two parts.

The first part of this paper is the very definition of the operations. In this aspect the paper is completely self-contained. The approach we use is to first introduce discretized versions of the topological operads and PROPs of [K5] and then to let these operate via correlation functions. In this completely cyclic setting it is more natural to define multilinear maps to $k$ rather than maps in $\operatorname{Hom}\left(V^{\otimes n}, V\right)$. The problem with this approach is that maps to $k$ are not easily composed which is why we introduce the notion of operadic correlation functions. The method of using correlation functions also mixes well with the ideas of physics where these objects are fundamental. Taking a physics perspective, anything which does not change a correlation function is not physical; in other words, OPEs live only inside correlators. In one of our main examples, namely that of a quasi-Frobenius algebra, this means that we can lift the constructions from the cohomology to the co-cycle level.

The second part is to show that this action has the desired operadic or PROPic properties. For this we use the operadic correlation functions to get the results on the discretized level. The last step, which is the one that requires the results of [K5], is to relate the operadic/PROPic structure of the discretized/graph level to the cell level operations of the chain model operads introduced in loc. cit. The relevant facts are reviewed in the first paragraph. The language we use is that of arc graphs on surfaces. This ties in with the description of the arc operad A Arc of [KLP]. In the special case of quasi-filling arcs, that is the subspace $\mathcal{A} r c_{\#}^{0}$ of $\mathcal{A} r c$ which corresponds to the moduli space $M_{g, n+1}^{1^{n+1}}$, there are actually two formalisms which one can use: the arc graphs and their dual ribbon graphs. We write out the details in both of these pictures, so that the reader more familiar with ribbon graphs can more easily understand the constructions. They are however different from the usual known constructions and they do not generalize to the boundary, that is to the more general, non-quasi-filling case, which is needed to define the string topology type operations.

When dealing with the operadic/PROPic properties, one has to be very careful about the operations on the side of the endomorphism operad $\mathscr{H o m}$ of the relevant linear spaces. This is a subtlety which is known from Deligne's conjecture. When being precise about the signs, one actually does not prove that one has an algebra over the relevant operad but rather an operadic morphism to the operad Brace which is formed by subspaces of the endomorphism operad, but has different sign rules and hence a twisted operad structure. When dealing with our actions, a similar situation arises which is slightly more complicated. We again obtain an operadic morphism to an operad which is formed by subspaces of the endomorphism operad. These spaces have a grading and the induced suboperad structure respects the associated filtration, but not the grading. Projecting to the associated graded operad structure and correcting the sign according to the grading, we obtain operadic actions as operadic morphisms 
to these "twisted" endomorphism suboperads.

The paper is organized as follows. In the first paragraph, we review the necessary facts we need from the first part and then define the "discretized" versions of the spaces we will consider. These "discretized" versions are free Abelian groups of graphs on surfaces, so-called partitioned arc graphs. We then define operad and PROP structures on these graphs and go on to show that partitioning an arc graph, which can be thought of as indexing a cell of an operadic cell complex, is an operadic morphism. This is actually quite subtle, since different types of graphs require different types of cell operads. The principal choices are filtered or graded versions. There is an intricate interplay between the discrete data associated to the graphs and the geometry they realize. In $\$ 2$ we define the notion of operadic correlation functions and give several examples including the action on the tensor algebra of a vector space. Paragraph $\S 3$ is dedicated to defining correlation functions, also known as correlators, for an all encompassing class of graphs, the angle marked partitioned arc graphs. These correlators are actually defined on the tensor algebra of a quasi-Frobenius algebra. In $\S 4$ we show that the correlators become operadic in several different settings. Notably for $\overline{\mathcal{A r c}}^{i \leftrightarrow o}$ and for $\mathcal{A} r c_{\#}^{0}$, whose definition is reviewed in $\$ 1.1 .5$ below. In the latter case, we have to be careful about the operadic structure of the spaces the operations take values in. The relevant subset of the Hom operad is graded and hence filtered. As mentioned above, the correlators define an operadic map to the associated graded of this filtration. A priori the operadic compositions in the Hom operad and the discretized graphs only agree up to lower order terms in the associated filtration. A posteriori these terms agree for the action of $\overline{\mathcal{A r c}}^{i} \leftrightarrow o$. The last paragraph $\S 5$ contains concluding remarks about the link to $D$-branes and future research directions.

Acknowledgments. We would like to thank the Max-Planck-Institute for Mathematics where this work was started, a good portion of it was written in the summer of 2005 and the finishing touches were put on in the summer of 2006. The two papers received their final form at the MSRI, which we would like to thank for its hospitality in May 2006. It is a pleasure to thank Bob Penner, Ralph Cohen, Jim McClure, Dev Sinha and Craig Westerland for discussions on various details during various stages of this project.

Conventions. We fix $k$ to be a field of arbitrary characteristic. Also in this part of the paper we always assume that the number of punctures is zero. That is $s=0$ for all operads and suboperads. 


\section{Discretizing the Arc operad and its cousins}

1.1. Brief review. Without going into the details, which are contained in [K5], we wish to point out the basic definitions of the graphs underlying the various versions and generalizations of the $\mathcal{A} r c$ operad. On a proper subset of $\mathcal{A} r c$, the quasi-filling arc families $\mathcal{A} r c_{\#}^{0}$ there are two pictures, one in terms of arc graphs and one in terms of the dual ribbon graphs. Although this subset is not big enough, even for our purposes - for instance to define the string topology type operations - we include both pictures, since ribbon graphs are commonly used to describe moduli spaces and are hence predominant in the literature.

1.1.1. Graphs. A graph is a tuple $\Gamma=\left(V(\Gamma), F(\Gamma), l_{\Gamma}, \partial_{G}\right)$ where $V(\Gamma)$ is a set whose elements are called the vertices, $F(\Gamma)$ is a set whose elements are called the flags or "half edges", $\imath_{\Gamma}: F(\Gamma) \rightarrow F \Gamma$ ) pairs the "half edges" to edges and $\partial_{\Gamma}: F(\Gamma) \rightarrow V(\Gamma)$ gives the vertex of a flag. An edge in this setting is an orbit of $l$ that is a set of flags $\{f, l(f)\}$. An oriented edge is a pair of flags $(f, l(f))$. The set of flags incident to a vertex $v$ is called $F_{v}(\Gamma)$.

Recall that a ribbon graph is a graph with a cyclic order of each of the sets of flags incident to a fixed vertex. Such a ribbon graph has natural bijections $\curvearrowright_{v}: F_{v} \rightarrow F_{v}$ where $\curvearrowright_{v}(f)$ is the next flag in the cyclic order. Since $F=U F_{v}$ one obtains a map $\curvearrowright: F \rightarrow F$. The orbits of the map $N:=\curvearrowright \circ l$ are called the cycles or the boundaries of the graph. These sets have the induced cyclic order. Due to the cyclic order a ribbon graph also can be "fattened" to a surface with boundary, by realizing the graph as a CW complex and then thickening the edges to bands. In this fashion one obtains a surface whose boundary components correspond to the cycles. The genus of such a graph is given by the genus of this surfaces. Explicitly, $2-2 g(\Gamma)=|V(\Gamma)|-|E(\Gamma)|+$ \#cycles. An $S$ marking of a ribbon graph $\Gamma$ is a bijection $\{\operatorname{cycles}(\Gamma)\} \rightarrow S$.

An angle is a pair of flags $(f \curvearrowright \curvearrowright(f))$, we denote the set of angles by $\angle_{\Gamma}$. It is clear that $f \mapsto(f, \curvearrowright(f))$ yields a bijection between $F_{\Gamma}$ and $\angle_{\Gamma}$. An angle marking by a set $T$ is a map $m k^{\angle}: \angle_{\Gamma} \rightarrow T$. We will call a (not necessarily connected) ribbon graph with an angle marking by $\mathbb{Z} / 2 \mathbb{Z}$ simply an angle marked ribbon graph.

1.1.2. Arc graphs. Fix an oriented surface $F=F_{g, r}^{s}$ of genus $g$, with $s$ punctures and $r$ labelled boundary components that each contain one marked point. We usually label the boundaries from 0 to $r-1$. An arc graph on $F$ is a particular class of graphs on $F$ whose vertices coincide with the marked points on the boundary. We frequently call these edges of these graphs "arcs". Since a vertex of the arc graph corresponds to a boundary component, the vertices are labelled. We will write $v_{i}$ for the vertex labelled by $i$, that is the unique vertex lying on the boundary $i$ of the surface.

There are certain other conditions on the graphs namely: there is at least one arc, there are no parallel arcs (by homotopy fixing the endpoints) and no arcs are parallel 
to a boundary component (again using a homotopy fixing the endpoint); see [K5], Section 2.1.1, for the full details.

More precisely, by [K5], Definition 2.1, a graph $\gamma$ on a surface is a triple $(F, \Gamma,[i])$ where $[i]$ is an equivalence class of embeddings of the realization of a graph $\Gamma$ into that surface. Here equivalence classes are taken under homotopies fixing the images of the vertices which are required to map surjectively to the marked points on the boundaries.

The pure mapping class group $P M C$ which fixes the marked points and punctures and otherwise fixes the boundaries setwise acts on each the classes $\gamma$ and gives rise to new equivalence class under this action. The equivalence class of $\gamma=(F, \Gamma,[i])$ under the action of $P M C$ denoted by $\bar{\gamma}$ and is called an arc graph. We will denote the isomorphism class of complementary regions by $\operatorname{Comp}(\bar{\gamma})$; that is the components of $F \backslash \gamma$ for any choice of representative $\gamma$ up to the bijections induced by changing the representative. We will also set $|\bar{\gamma}|=\left|E_{\Gamma}\right|$.

Fixing $F$, the main classes of graphs and subclasses are:

\begin{tabular}{|l|l|l|}
\hline Graphs & Name & Definition \\
\hline$\overline{\mathscr{g}}$ & arc graphs & $\begin{array}{l}\text { equivalence classes of graphs under } \\
P M C \text { action }\end{array}$ \\
\hline$\overline{\mathscr{g}}^{e}$ & exhaustive & there are no vertices with valence 0 \\
\hline$\overline{\mathscr{g}}_{\#}$ & quasi-filling & $\begin{array}{l}\text { the complementary regions of the arcs are } \\
\text { at most once-punctured polygons }\end{array}$ \\
\hline
\end{tabular}

1.1.3. Inner and outer angles, twisted arc graphs, and Ins and Outs. An arc graph has a linear order of all the flags at a vertex due to the induced orientation of the surface and hence a total order on all flags, by first enumerating the flags according to their labelled boundary components and then according to their linear order at that component. The last and first flag at a vertex form a distinguished angle which is called the outside angle at that vertex. All angles besides the outside angles are called inner angles.

An arc graph is called twisted at the boundary $i$ if the first and last arc incident to $i$ are homotopic in $F$, when one allows homotopies that move the endpoint on the boundary component $i$.

We will also consider arc graphs in which the set of boundaries of the surface is partitioned into the sets In and Out. This partitioning is encoded in a map $i / o: V(\Gamma) \rightarrow \mathbb{Z} / 2 \mathbb{Z}$, where the value 1 stands for "in" and 0 stands for "out". Recall that the set of vertices of the arc graph can naturally be identified with the boundary components of the surface. 
1.1.4. Dual ribbon graphs. For a graph in $\overline{\mathscr{g}}_{\#}$ there is a natural dual ribbon graph. We will briefly review the case with no punctures $s=0$. The case with punctures is treated in [K5], Section 3.3.3. The map LLoop of [KLP] gives a generalization of the dual graph to the non-quasi-filling case. This is important for the stabilization of [K6].

By using the orientation on the surface an arc graph is actually a (possibly disconnected) ribbon graph. To this we associate a dual graph which is a marked ribbon graph (see Definition 1.1) that is defined as follows: Choose a representative $\gamma$, decompose the surface into the complementary regions. Now associate a vertex to each complementary region and an edge to each arc. Each edge connects the vertices (or vertex) representing the regions on the two sides of the arc the edge represents. This graph is again a ribbon graph, by using the orientation of the arc graph to give the arcs bordering a polygonal complementary region a cyclic structure. Each cycle of this dual ribbon graph corresponds to a boundary component of the surface and hence has a linear order. That is for each cycle there is a distinguished flag which is the first in this cycle.

Vice versa, we can "fatten" a given marked ribbon graph to a surface and consider the graph as the spine of this surface. Applying a dual graph construction in this setting produces an inverse to the construction of the dual graph of an arc graph.

Note that in the dual ribbon graph picture, "in" and "out" are marked by a labelling of the cycles by $\mathbb{Z} / 2 \mathbb{Z}$.

1.1.5. Spaces of graphs. We obtain the space of a given class of graphs by looking at the set of projective metrics, that is equivalence classes of maps $w_{\Gamma}: E(\Gamma) \rightarrow \mathbb{R}_{>0}$ under the action of $\mathbb{R}_{>0}$ by a global rescaling; that is the action given by $\lambda \in \mathbb{R}_{>0}$ : $(\lambda w)(e)=\lambda w(e)$. The set of all graphs of a given class with projective metric basically gets a topology by identifying the limit in which $w(e) \rightarrow 0$ for some edge with the graph in which $e$ is deleted. We usually call elements of these spaces projectively weighted arc families in keeping with [KLP] and the work of Penner.

The most important spaces are:

- $A r c_{\#}^{0}$ the space defined by quasi-filling graphs. This space is isomorphic to $M_{g, n+1}^{1^{n+1}}$, the moduli of curves of genus $g$ with $n$ marked points and one tangent vector at each of these points.

- $\overline{\mathcal{A r c}}^{i \leftrightarrow o}$ the space of arc graphs with a projective metric, together with a partitioning $i / o$ into In and Out, which satisfy the following conditions: (1) there are only arcs between "in" and "out"; (2) each "in" boundary vertex has valence at least 1 . This space plays the role of Sullivan chord diagrams.

A reference list of the spaces that will make an appearance are:

- $A$ the space of all arc graphs with a projective metric. This is a CW complex whose cells are indexed by the arc graphs. 
- Arc the subspace of all exhaustive arc graphs with a projective metric.

- $\mathcal{A}^{<}$the space of all elements of $\mathcal{A}$ with an additional angle marking.

- $A r c^{<}$the space of all the exhaustive arc graphs together with a projective metric and an angle marking by $\mathbb{Z} / 2 \mathbb{Z}$. We will consider $\mathcal{A} r c$ as a subspace of $\mathcal{A} r c^{L}$ by choosing the constant marking $m k^{L} \equiv 1$.

- $\mathcal{A}^{i / o}$ the space of arc graphs with projective metric which have an additional marking $i / o$ of the boundaries distinguishing inputs and outputs. We will consider this space again as a subspace of $\mathcal{A}^{<}$by marking all outside angles and all inner angles of the In boundaries by 1 while marking all inner angles of the Out boundaries by 0 .

- $\mathscr{A}^{i \leftrightarrow o}$ the subspace of $\mathscr{A}^{i / o}$ which is comprised of the arc graphs with a projective metric that additionally satisfy the condition that there are only arcs between In boundaries and the Out boundaries.

- $A r c^{i \leftrightarrow o}$ the subspace whose underlying arc graphs are exhaustive and all of whose arcs only run from In boundaries to Out boundaries.

- $\bar{A} \boldsymbol{A r c}^{i \leftrightarrow o}$ the subspace of $\mathscr{A}^{i \leftrightarrow o}$ whose underlying arc graphs hit all the In boundaries.

The spaces above naturally come as disjoint union over the number of boundary components, which we usually think of as labelled by $\{0, \ldots, n\}$. In the case of $\mathcal{A}^{i / o}$ we first label the boundaries and In and Out, and then label these boundaries separately, say, by $\{1, \ldots, n\}$ and $\{1, \ldots, m\}$. There are natural actions of the permutation groups on these labels. In the first part, we showed essentially that $\mathcal{A}^{<}$and its subspaces are operads and that $\overline{\mathcal{A r c}}^{i \leftrightarrow o}$ is a quasi-PROP. Actually some of these spaces, notably $\mathcal{A} r c_{\#}^{0}$, are only rational operads, viz. defined on an open dense set. The full details are quite elaborate and make up the bulk of [K5].

1.1.6. Operads/PROPs of arc graphs. Each of these spaces has an associated graph complex cell model given by considering the free Abelian groups generated the underlying graphs. The natural differential is given by restricting the differential of the $\mathrm{CW}$ complex $\mathcal{A}$. This differential applied to a graph is the sum of the arc graphs obtained by removing one arc with the appropriate sign. The differential for the open cells of a subspace is defined to be the sum over only those graphs which correspond to elements in the subspace. In other words these complexes are the relative complexes of the subspaces in $\mathcal{A}$. These complexes inherit algebraic operad and/or PROP structures by treating the graphs as "open cells". Here an open cell is constituted by elements corresponding to the possible projective metrics of a fixed arc graph, and we label the generator corresponding to an open cell by the respective graph. Then there are induced gluing operations from the topological level on the "open cells", see [K5], Section 2.3.2. These cells are graded by their dimension, which is the number of edges 
of the graph minus one. We note that the number of edges is also twice the number of flags, which coincides with the number of angles. The gluing operations respect the filtration induced by the grading and accordingly we obtain two versions of cell operads on the graph complex. The first is the induced structure on the "open cells" which we denote by $\ell_{o}^{*}(\cdot)$, and second one is the one induced by the first structure on the associated graded of the filtration by dimension. The latter is again of course additively isomorphic to the former and both are isomorphic to the graph complex. The operations differ however. To make this distinction clear we denote the graph complexes with the operations corresponding to the associated graded by $\operatorname{Gr} \ell_{o}^{*}(\cdot)$. On the cell level possibly after passing to the associated graded, we always obtain the honest structure, which is not the up to homotopy or a rational version. Most importantly:

- The associated graded cell complex $\operatorname{Gr} \mathcal{C}_{o}^{*}\left(\mathcal{A} r c_{\#}^{0}\right)$ is a dg operad and $\operatorname{Gr} \mathcal{C}_{o}^{*}\left(\mathcal{A} r c_{\#}^{0}\right)$ calculates the cohomology of $M_{g, n+1}^{1^{n+1}}$. By using the angle marking $m k^{<} \equiv 1$ the operad $\operatorname{Gr} \mathcal{C}_{o}^{*}\left(\mathcal{A} r c_{\#}^{0}\right)$ embeds into $\mathrm{Gr} \mathcal{C}_{o}^{*}\left(\mathcal{A} r c^{<}\right)$.

- The cell complex $\ell_{o}^{*}\left(\overline{\mathcal{A} r C}^{i \leftrightarrow o}\right)$ is a PROP and Gr $\ell_{o}^{*}\left(\overline{\mathcal{A r C}}^{i \leftrightarrow o}\right)$ isomorphic to the cellular chains $C C_{*}\left(\overline{\mathcal{A r c}}_{1}^{i \leftrightarrow o}\right)$ of a CW-complex $\overline{\mathcal{A r}}_{1}^{i \leftrightarrow o}$, and these chains have the structure of a dg-PROP. This PROP can be thought of as the PROP of Sullivan chord diagrams.

The details are quite involved, and they are carefully spelled out in [K5].

\subsection{Partitioned ribbon graphs}

1.2.1. Inserting points into edges. To define the operations on the Hochschild cochains, we will systematically deal with unstable graphs, i.e., graphs which have vertices of valence two. For this we will need the operation of inserting vertices of valence 2 into edges and also the reverse operation of removing them.

First, we recall the notion of a marked ribbon graph, viz. the type of stable graphs we consider.

Definition 1.1. A marked ribbon graph is a ribbon graph together with a map $\mathrm{mk}:\{$ cycles $\} \rightarrow F_{\Gamma}$ satisfying the conditions:

i) For every cycle $c$ the directed edge $\mathrm{mk}(c)$ belongs to the cycle.

ii) All vertices of valence two are in the image of $\mathrm{mk}$, that is, for all $v$, $\operatorname{val}(v)=2$ implies that $v \in \operatorname{Im}(\partial \circ \mathrm{mk})$.

We called the set and the Abelian group generated by these graphs $\mathcal{R} i b$. It is naturally the disjoint union over the graphs $\mathcal{R} i b(n)$ which have $n$ cycles. We showed in the first part that if we think of $\mathcal{R} i b$ as labelled graphs, they form an operad by 
inducing said structure via the isomorphism $\mathcal{R} i b \cong \mathrm{Gr} \mathcal{C}_{o}^{*}\left(\mathcal{A} r c_{\#}^{0}\right)$ induced by the dual graph construction.

Definition 1.2. Given a graph $\Gamma$ and an edge $e=\left\{f_{1}, f_{2}\right\} \in \Gamma$, we let $\Gamma\left(e^{\prime}\right)$ be the graph whose vertices are $V_{\Gamma} \amalg\left\{v_{2}\right\}$ with flags $F_{\Gamma} \amalg\left\{n_{1}, n_{2}\right\}$ such that $\partial\left(n_{i}\right)=v_{2}$ and $l\left(f_{i}\right)=n_{i}$. We say $\Gamma\left(e^{\prime}\right)$ is obtained from $\Gamma$ by inserting a vertex into $e$. Notice that this insertion does no disturb the cycles, that is, there is a canonical identification of the cycles before and after the insertions. If $\Gamma$ has a marking on its cycles this marking will simply be retained.

Vice versa, if $v_{2}$ is a vertex of valence 2 with flags $n_{1}, n_{2}$ such that $l\left(n_{i}\right)=f_{i}$, then we let $\Gamma / v_{2}$ be the graph whose vertices are $V_{\Gamma} \backslash\left\{v_{2}\right\}$, whose flags are $F_{\Gamma} \backslash\left\{n_{1}, n_{2}\right\}$ and whose new relation for $l$ is $l_{\Gamma / v_{2}}\left(f_{1}\right)=f_{2}$. In case $n_{i}=\operatorname{mk}(c)$ for the cycle $c$ it lies on, we set $\mathrm{mk}_{\Gamma \backslash v_{2}}=(n \circ \imath)_{\Gamma}^{-1}\left(n_{i}\right)$.

We write $\Gamma^{\prime} \triangleright \Gamma$ if $\Gamma$ is obtained from $\Gamma$ by repeatedly inserting vertices, i.e., if there is a sequence $\Gamma^{0}, \ldots, \Gamma^{n}, \Gamma^{i}=\Gamma^{\prime i-1}(e)$ for some $e \in E_{\Gamma^{i-1}}$, and $\Gamma^{\prime}=\Gamma^{n}$, $\Gamma=\Gamma^{0}$.

Definition 1.3. A partitioned marked ribbon graph is a ribbon graph together with a map mk: $\{$ cycles $\} \rightarrow F_{\Gamma}$ which satisfies the condition that for every cycle $c$ the flag $\mathrm{mk}(c)$ belongs to that cycle.

We let $V_{2}(\Gamma)=\left\{v \in V_{\Gamma} \mid \operatorname{val}(v)=2\right\}$ be the vertices of valence two and set $V_{\text {part }}=V_{2} \backslash \operatorname{Im}(\partial \circ \mathrm{mk})$ to be the partitioning vertices.

Notation 1.4. Let $\mathcal{R} i b^{\text {part }}$ be the set of all partitioned marked ribbon graphs. To avoid cluttered notation, following [K5], we abuse notation and denote by $\mathcal{R} i b^{\text {part }}$ the set of graphs, the Abelian group generated by it, as well as the collection $\left\{\mathcal{R} i b^{\text {part }}(n)\right\}$ where $\mathcal{R} i b^{\text {part }}(n)$ is the Abelian group generated by the subset of $\mathcal{R} i b^{\text {part }}$ of graphs which have $n+1$ cycles that are labelled by $\{0, \ldots, n\}$ together with the $\mathbb{S}_{n+1}$ action permuting these labels. The various meanings will always be clear from the context.

For a marked ribbon graph $\Gamma$ we will consider $\mathcal{P}: \mathcal{R} i b \rightarrow \mathcal{R} i b^{\text {part }}$,

$$
\mathcal{P}(\Gamma)=\sum_{\Gamma^{\prime} \triangleright \Gamma} \pm \Gamma^{\prime},
$$

and call it the partitioning of $\Gamma$. The right-hand side is infinite, but it is graded by the number of partitioning vertices $\left|V_{\text {part }}\right|$. The fastidious reader hence may take the $\mathcal{P}(\Gamma)$ to lie in $\mathcal{R} i b^{\text {part }}[[t]]$, where $t$ is a variable whose power corresponds the number of partitioning vertices. The sign is explained in §1.3.4.

Remark 1.5. This construction can be seen as the generalization of the foliage operator of [K4], [K2] from the setting of treelike ribbon graphs to the setting of general ribbon graphs. 
1.3. Discretizing the A $\boldsymbol{A} c$ operad. Given a tuple $\alpha=(F, \Gamma, \overline{i i}])$, we will consider a series of embedded graphs which are obtained by drawing parallel arcs.

Notation 1.6. Let $p=\left(n_{1}, \ldots, n_{k}\right)$ be an ordered partition of $n \in \mathbb{N}$ with each $n_{i}>0$, and denote the set of all these partitions by $P(n, k)$.

Let $\alpha \in \overline{\mathscr{g}}^{e}$. Recall that all edges $E(\alpha)=E_{\Gamma(\alpha)}$ are linearly ordered by enumerating the flags in the following order: first according to their boundary and then according to the linear order at that boundary component induced by the orientation of the surface. Let $k=|E(\alpha)|$ and $p \in P(n, k)$. We define $\alpha^{p}$ to be the embedded graph obtained from $(F, \Gamma, \overline{[i]})$ by inserting $n_{i}-1$ parallel edges to the $i$-th edge $e_{i}$ and embedding them parallel to $e_{i}$. We call the result of this operation a partitioned arc graph and denote the set of these by $\mathcal{P} \overline{\mathcal{E}}^{e}$. And again we use the conventions explained in $\S 1.4$.

1.3.1. Drawing arc graphs. An example of such a graph is given in Figure 1. In

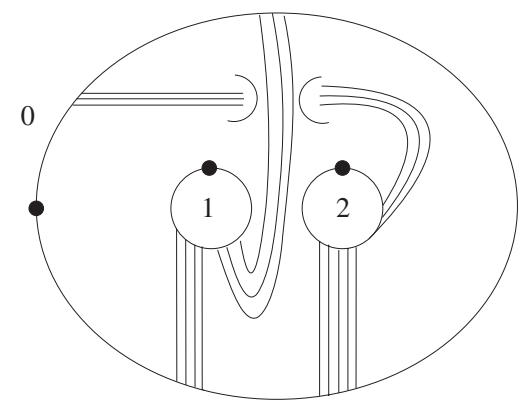

Figure 1. A partitioned arc graph for the partition $(4,5,3,3)$.

this figure and in all other figures, we have taken the liberty to depict the arc graphs in a more suitable way. By definition, all edges of a graph are incident to the vertices, which would clutter the pictures. We therefore move the endpoints along the boundary component that they are incident slightly apart. This is done in such a fashion that they (1) all are distinct and distinct from the original vertex, and (2) their linear order along the boundary component starting at the original vertex coincides with their original linear order. We could have even defined the arc graphs in this manner; see, e.g., [KLP] for all topologically equivalent ways to define the space $\mathcal{A}$. We will therefore henceforth use both pictures: the one with the edges apart as in Figure 1 and the true graph picture, where all the edges are incident to their vertices. The advantage of the latter lies in the more direct definition, and the advantage of the former is twofold: first one obtains nicer pictures and secondly, the boundaries of the complementary regions are $2 k$-gons whose sides alternatingly correspond to boundary components 
and arcs. This last observation will make the definition of the action of these graphs on the Hochschild co-chains more transparent.

We define

$$
\mathcal{P}(\alpha)=\sum_{n \geq k} \sum_{p \in P(n, k)} \pm \alpha^{p} .
$$

As always when dealing with this type of object, one can use a formal variable $t$ to keep track of the total number of edges and consider the expression of equation (1.2) as a formal power series in $\mathbb{P} \mathcal{R} i b[[t]]$. The sign is explained in $§ 1.3 .4$.

1.3.2. The underlying arc graph. Given a partitioned arc graph $\gamma \in \mathcal{P} \overline{\mathscr{E}}$ it is possible to recover a unique $\alpha \in \overline{\mathscr{E}}$ such that $\gamma$ is a summand on $\mathcal{P}(\alpha)$. Namely, for $\gamma \in \mathcal{P} \overline{\mathscr{E}}$, we define the underlying arc graph $\bar{U}(\gamma)$ to be the graph obtained by gathering all parallel edges into one edge. Formally we introduce an equivalence relation on $E(\gamma)$ where $e_{1} \sim e_{2}$ if $e_{1}$ and $e_{2}$ are parallel - as arcs in the surface via a homotopy fixing the endpoints - and then take the quotient by this relation. This is well defined since if $e_{1} \sim e_{2}$ then $\partial\left(e_{1}\right)=\partial\left(e_{2}\right)$.

We call a partitioned arc graph quasi-filling if the complementary regions are either polygons or once-punctured polygons. Notice that a partitioned arc graph is quasi-filling if and only if its underlying arc graph is quasi-filling.

1.3.3. Grading. Let $\mathcal{P} \overline{\mathscr{E}}^{e}(n)^{k}$ be the set of those graphs whose underlying arc graph has $k+1$-edges which "live" on surfaces with $n+1$ boundaries labelled from $\{0, \ldots, n\}$. We let $\mathcal{P} \overline{\mathscr{E}}^{e}(n):=\bigoplus_{k} \mathcal{P}_{\overline{\mathscr{E}}}^{e}(n)^{k}$. This space is then graded by $k$ and hence filtered by the elements of degree $\leq k$.

Remark 1.7. We can view $\mathcal{P} \overline{\mathscr{E}}^{e}$ as a discretized version of $\mathcal{A} r c$ in the following two ways: $\mathcal{P}(\alpha)$ can be thought of as either (A) as a sampling by the numbers $\frac{i}{k}$ of the boundary considered as the interval defined by the window on the boundary (see [KLP], [KP] for the formalism of windowed surfaces) or equivalently (B) as a cosimplicial realization of the arc graph $\alpha$. We wish to pursue the latter point of view elsewhere (see also the comments $§ 5$ below).

1.3.4. Signs. In this paragraph, we wish to fix our sign conventions once and for all. As in [K4], we do this by using tensor products indexed by sets in the spirit of [KS1], which makes all signs completely natural. We will henceforth not bother with them again. For other different explicit sign fixing schemes for operations of cell operads, we refer to [K4].

First notice that for any arc graph partitioned or not, there is a natural linear order on all the flags and hence on all the edges. So we can use these linear orders to fix the signs. In general one can do this quite nicely by considering the tensor product over $\mathbb{Z}$ of the generator given by the graphs $\alpha$ with copies of a "line of degree 1 " that 
is a freely generated Abelian group generated by an element of degree 1 . Thus we replace $\alpha$ with the expressions $\alpha \otimes L^{\otimes S}$ where $S$ is an indexing set. In the cyclic operad setting we will use $S=E^{\prime}(\Gamma)$, the set of edges without the last edge in the linear order. This gives a universal way to fix the signs. It also assigns the correct degree to $\alpha$ if $\alpha$ is thought of as an element of the various cellular chain complexes introduced in [K5]. For the expression above this results in the sign obtained from the shuffle $L^{\otimes E^{\prime}(\alpha)} \otimes L^{\otimes n}$ to $L^{\otimes E^{\prime}\left(\alpha^{p}\right)}$ for the summand indexed by $p \in P(n, k)$.

In the PROP setting the natural indexing set will be $S=\angle_{\text {inner }}^{\text {in }}(\Gamma)$ the set of inner angles on the inputs. This again corresponds to the dimension of the cells when we consider the CW complex $\overline{A A r c}^{i \leftrightarrow o}$.

Of course one of the two sign conventions can be obtained from the other by shifting the complexes. Alternatively, one could grade by shifting by the dimension of the corresponding spaces to get rid of the signs for top dimensional cells, as was done to obtain the Hopf algebra of Connes and Kreimer in [K4]. Or one could shift by the number of all the boundaries. In this vein, we can consider the use of $E^{\prime}$ as the shift from the grading of $\mathfrak{D A} r c$ by a line associated to the operadically distinguished boundary 0 .

1.4. Compatibility of the two constructions in the quasi-filling case. Recall that for a quasi-filling arc graph $\Gamma$ we defined a dual ribbon graph $\hat{\Gamma}$ in [K5] as outlined in $\S 1.1$. This construction easily generalizes to quasi-filling $\Gamma \in \mathcal{P} \overline{\mathscr{g}}^{e}$. Moreover, since we are simply inserting parallel edges, on can see that $\Gamma \in \mathcal{P} \overline{\mathscr{E}}^{e}$ is quasi-filling if and only if $\Gamma$ is a summand of $\mathcal{P}\left(\Gamma^{\prime}\right)$ for some quasi-filling arc graph $\Gamma^{\prime}$.

Lemma 1.8. Denoting the dual ribbon graph of a quasi-filling (partitioned) arc graph $\alpha$ by $\hat{\Gamma}(\alpha)$ and extending this construction linearly to give a map of the respective Abelian groups, the following equality holds:

$$
\hat{\Gamma}(\mathcal{P}(\alpha))=\mathcal{P}(\hat{\Gamma}(\alpha)) .
$$

Proof. The insertion of parallel edges corresponds to adding rectangles into the set of complementary regions and this corresponds to inserting vertices into the edges of the dual ribbon graph.

For an example of a partitioned graph and its dual see Figure 2.

1.4.1. The operad structure. The set of Abelian groups generated by the $\mathcal{P} \overline{\mathscr{E}}(n)$ has a natural cyclic operad structure, where the $\mathbb{S}_{n+1}$ action is given by permutation of the boundary labels.

Given two elements $\alpha \in \mathcal{P} \overline{\mathscr{G}}(m)$ and $\beta \in \mathcal{P} \overline{\mathscr{G}}(n)$, we will call them matched at the boundaries $i$ and 0 if the number of arcs incident to $\alpha$ at the boundary $i$ is equal to the number of arcs incident to $\beta$ at the boundary 0 . In case $\alpha$ and $\beta$ are matched at 

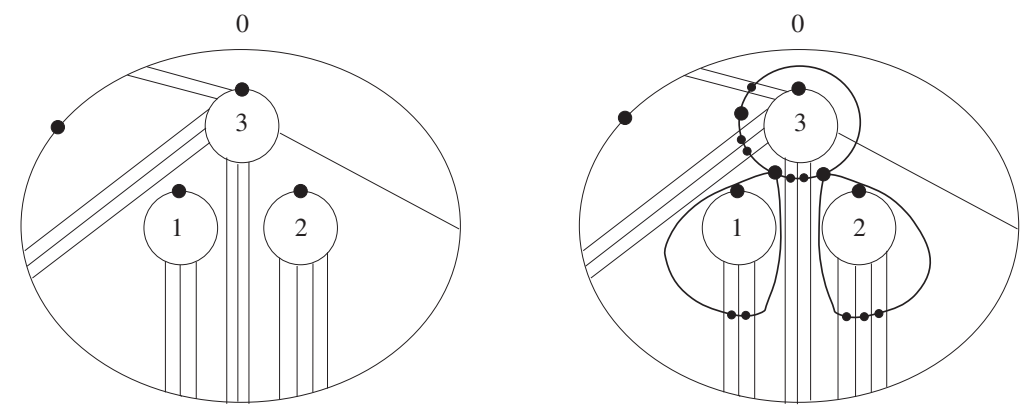

Figure 2. A quasi-filling partitioned arc graph and it dual partitioned marked ribbon graph.

the boundaries $i$ and 0 , we define $\alpha \circ_{i} \beta$ to be the graph obtained by gluing the edges of $\alpha$ incident to $i$ to those of $\beta$ incident to 0 matching them according to their linear order. As usual this means that we reverse the linear order at $i$, that is, read off last to first, and match this order with the linear order on 0 . If there is no matching at the boundaries, we set $\alpha \circ_{i} \beta=0$. There are two final steps:

(1) if both $\alpha$ and $\beta$ are twisted at their boundaries 0 and $i$, we set their composition zero, and

(2) if there are any closed loops, that is, embedded arcs that do not touch any boundary as edges, we set the product zero.

If we omit step (1) we also call the gluing the algebraic gluing and in contrast call the gluing with both steps (1) and (2) the geometric gluing; see $\$ 1.19$ for comments.

It is clear that this gives an operad structure and that the subset $\mathcal{P} \overline{\mathscr{E}}^{e}(n) \subset \mathcal{P} \overline{\mathscr{E}}(n)$ of exhaustive graphs is a suboperad.

Lemma 1.9. The map $\mathcal{P}$ is an operadic morphism; that is, we have the following formula for the compatibility between the partitioning of arc families and the operad compositions:

$$
\mathcal{P}\left(\alpha \circ_{i} \beta\right)= \pm \mathcal{P}(\alpha) \circ_{i} \mathcal{P}(\beta)
$$

for $\alpha, \beta \in \overline{\mathscr{E}}$ where the sign is the sign discussed in $\$ 1.3 .4$.

Proof. This is simply the observation that first discretizing and then gluing corresponds to the same combinatorics as first gluing and then discretizing. This fact becomes clear if one cuts the graphs occurring on the right-hand side along the simple separating curve which corresponds to the image of the two glued boundaries. If there are no closed loops and not both of the boundaries are twisted, the result is immediate. If both of the boundaries are twisted this would yield 0 in the open cell cellular chains of $\mathcal{A}$ and hence on $\overline{\mathscr{E}}$ by definition. We see, however, that it is not possible to set 
them zero and discretize them. This would again result in the gluing of discretized twisted arc graphs, so that the condition of being twisted in the partitioned case and the non-partitioned case agree, and in both cases the relevant contributions are set zero. The same reasoning applies when we erase closed loops. See $\$ 2.3$ of [K5] for the definition of the open cell gluing.

1.4.2. Self-gluing, modular operads and PROPs. In the above procedures for gluing partitioned arc graphs, we do not have to assume that the two boundaries we glue actually lie on the same surface.

Proposition 1.10. Allowing self-gluing, the gluing operations of $\S 1.4 .1$ turn $\mathcal{P} \overline{\mathscr{E}}(n)$ into a modular operad. Here the additional modular grading variable " $g$ " is given by the genus $g .{ }^{2}$

1.4.3. Partitioned graphs with In and Out markings. Just like for arc graphs, we can look at partitioned arc graphs together with a $\mathbb{Z} / 2 \mathbb{Z}$ marking of their boundaries, viz. a partitioning of their cycles into In and Out.

Notation 1.11. To avoid introducing yet other symbols for the classes of graphs indexing the cells of the different subspaces of $\mathcal{A}$, we simply denote the partitioned graphs by using the prefix $\mathcal{P}$, e.g., $\mathcal{P} \overline{\mathcal{A} r c}^{i \leftrightarrow o}, \mathcal{P} \mathcal{A} r c^{i \leftrightarrow i}$ and $\mathcal{P} \mathcal{A} r c_{\#}^{0}$.

Proposition 1.12. Restricting the modular operad structure and iterating it by gluing all In boundaries to all Out boundaries of two collections of elements of $\mathcal{P} \mathcal{A}^{i / o}$ impose a PROP structure on $\mathcal{P} \mathcal{A}^{i / o}$.

Also, similarly, there is a PROP structure - which we call the algebraic PROP structure - imposed on $\mathcal{P} \mathcal{A}^{i / o}$ which is obtained from the definition of the gluing $\S 1.4 .1$ by omitting the final step $(1) .^{3}$

Finally, the map $\mathcal{P}$ is operadic, or in the PROP case is PROPic.

Proof. The conditions of associativity are again straightforward if one cuts the glued surface in two different ways. The operadic properties are verified as above.

1.4.4. Partitioning angles, grading and the preservation of the filtration. An angle is a partitioning angle if its two edges, $e_{1}=\{f, l(f)\}$ and $e_{2}=\{\curvearrowright(f), l(\curvearrowright(f)\}$, are parallel. In the opposite case we call it non-partitioning.

With this definition, we can rewrite the grading of $\mathcal{P} \overline{\mathscr{E}}$ as given by half the number of non-partitioning angles minus one. The number of non-partitioning angles is precisely the number of edges of the underlying arc graph.

\footnotetext{
${ }^{2}$ We do not enforce the stability condition $3 g-3+n \geq 0$.

${ }^{3}$ The situation of step (2) actually never occurs.
} 
Furthermore, it is clear that the degree in the composition in $\mathcal{P} \overline{\mathscr{E}}^{e}$ goes down by one each time two non-partitioning angles (other that the outside angles) are glued, as this will decrease the number of non-partitioning angles by two. In all the other cases - gluing partitioning to partitioning and non-partitioning to partitioning - the number of partitioning angles is preserved. Lastly, erasing closed loops also only decreases the number of partitioning angles.

Therefore the following holds:

Lemma 1.13. The filtration given by the degree $\leq k$ graded components on $\mathcal{P} \bar{g}^{e}$ is respected by the gluings $\circ_{i}$. Moreover, the filtration is respected for all gluings in $\mathcal{P} \overline{\mathscr{E}}$ which do not glue an empty boundary to an empty boundary.

1.4.5. Graded version. We also have the same type of statement as in Lemma 1.9 in the graded case. Recall that we have a grading on $\mathcal{P} \overline{\mathscr{E}}^{e}$ and one on $\overline{\mathscr{E}}^{e}$, the latter is graded by the dimension of the cells which is the number of edges -1 . This grading is of course respected by $\mathcal{P}$. Also, in both cases the induced filtration is respected, so we get an operad structures on the associated graded.

Corollary 1.14. The map $\mathcal{P}$ of 1.9 induces an operadic morphism of the associated graded objects $\mathcal{P}: \operatorname{Gr} \mathcal{C}_{o}^{*}(\mathcal{A} r c) \rightarrow \mathrm{Gr} \mathcal{P} \overline{\mathcal{E}}^{e}$. Moreover, the same holds true for all suboperads, di-operads or PROPs whose compositions do not include a gluing of an empty boundary to an empty boundary.

Remark 1.15. Notice that in this graded version all the contributions from the gluing, which involve deleting closed loops, are set zero. This is true for both sides since deleting a closed loop decreases the grading. Moreover if the condition of step (1) is met, that is, if both boundaries are twisted, the gluing procedure of the algebraic gluing also decreases the grading, so that the associated graded of the topological gluing and the algebraic gluing agree.

1.4.6. Angle marked partitioned graphs. We will also consider the constructions of the last paragraphs in the case of angle marked graphs. An angle marked partitioned arc graph is a partitioned arc graph with an angle marking.

We let $\mathcal{P} \angle \overline{\mathscr{g}}(n)^{k}$ be the angle marked partitioned arc graphs on a surface with $n+1$ boundaries labelled by $\{0, \ldots, n\}$ whose underlying graph has $k+1$ edges. Put $\mathcal{P} \angle \overline{\mathscr{G}}(n)=\bigoplus_{k} \mathcal{P} \angle \overline{\mathscr{G}}(n)^{k}$. Then $\mathbb{S}_{n+1}$ acts by permutation on the labels, and we call the collection of $\mathbb{S}_{n+1}$-modules $\mathcal{P} \angle \overline{\mathscr{E}}$ simply $\mathcal{P} \angle \overline{\mathscr{E}}$. Again, we use the same notation for the set and the free Abelian group generated by it. Also keeping the standard notational conventions, we call $\mathcal{P}^{\angle \bar{g}^{e}}$ the subset/subgroup of exhaustive partitioned arc graphs. These spaces have a grading by $k$ and hence an induced filtration by elements of degree $\leq k$. 
Analogously to $\mathcal{P}$ there is a partitioning operator for angle marked arc graphs. Keeping the notation $a^{p}$ for a particularly partitioned graph as in $\S 1.3$, set

$$
\mathcal{P}\left(\alpha, m k^{<}\right)=\sum \pm\left(a^{p}, m k_{\alpha^{p}}^{<}\right)
$$

where $\left(m k^{L}\right)^{p}$ is the angle marking which marks every new partitioning angle by 1 and keeps the other markings of $\alpha$.

If $\alpha \in \mathcal{C}_{o}^{*}\left(\mathcal{A}^{i / o}\right)$, where we identify the cell with the arc graph, then $\alpha$ has a standard angle marking [K5] defined by marking all outside angles and all angles at the boundaries In by one and the rest, that is, the inner angles at the boundaries Out, by 0 . Likewise there is a standard marking for arc graphs $\alpha \in \bar{g}=C C_{*}(\mathcal{A})$, which is simply the constant marking by 1 . For an $\alpha$ of one of the two types above, we denote $\alpha$ together with its standard angle marking by $\alpha^{\perp} \in \overline{\mathscr{g}}^{<}$, and define

$$
\mathcal{P}^{\angle}(\alpha):=\mathcal{P}\left(\alpha^{\angle}\right) \text {. }
$$

Using the rationale of [K5], we identify a relative cell $\alpha \in \mathcal{C}_{o}^{*}\left(\mathcal{A} r c^{i / o}\right)$ with the angle marked arc graph $\alpha^{<}$that labels a cell of $\mathcal{A}^{<}$, the CW-complex of angle marked arc graphs.

Notation 1.16. Extending the Notation 1.11 we denote the embedding of a class of partitioned graphs into $\mathcal{P} \angle \overline{\mathscr{E}}$ by the prefix $\mathcal{P}^{<}$, viz. $\mathcal{P}<\overline{\mathcal{A} r c}^{i \leftrightarrow o}, \mathcal{P}^{\perp} \mathcal{A} r c^{i \leftrightarrow i}$ and $\mathcal{P}^{\circ} \mathcal{A} r c_{\#}^{0}$.

For the gluing we will need a new matching condition. Given an angle marking, we partition the set of flags $F=F(v)$ at a given boundary into subsets $F=F_{1} U$ $\cdots \amalg F_{k+1}$, where $k$ is the number of markings by 1 by collecting all the flags between which the angle marking is zero. Notice that $F$ has a linear order and we also think of the subsets as linearly ordered.

Definition 1.17. Two angle marked partitioned arc graphs $\left(\alpha^{p}, m k^{<}\right)$and $\left(\alpha^{\prime p}, m k^{L^{\prime}}\right)$ are angle matched at the boundaries $i$ and $i^{\prime}$ if the number of angles with an angle marking 1 agree for these two boundaries. We say that an angle marked partitioned arc graph is twisted at the boundary $i$ if the underlying arc graph has this property and each of the two edges forming the outer angle in the underlying graph has at least one more parallel edge.

In case two angle marked arc graphs are angle matched at the boundaries $i$ and $i^{\prime}$ there are equally many sets, say $k$, in the partitions of $F\left(v_{i}\right)$ and $F\left(v_{i^{\prime}}\right)$. We say that the graphs are perfectly angle matched if $\left|F\left(v_{i}\right)\right|>1$ implies that $\left|F\left(v_{i^{\prime}}\right)_{k-i}\right|=1$, and $\left|F\left(v_{i^{\prime}}\right)\right|>1$ implies that $\left|F\left(v_{i}\right)_{k-i}\right|=1$.

We will give the rigorous combinatorial definition of the gluing below. Geometrically, we move the edges on the boundaries slightly apart if the angle marking between 
them is 1 . Now the number of endpoints of these edges on the boundaries which are to be glued will coincide precisely if they are angle matched. In this case, we want to identify these vertices. The condition of perfect matching ensures that at any given vertex, there is at most one side which has more than one vertex.

Definition 1.18. For angle marked arc families $\left(\alpha^{p}, m k^{<}\right)$and $\left(\alpha^{p \prime}, m k^{L^{\prime}}\right)$ which are perfectly angle matched at the boundaries $i$ and 0 , we define $\left(\alpha^{p}, m k^{L}\right) \circ_{i}\left(\alpha^{\prime p}, m k^{L^{\prime}}\right)$ as follows. Let $F_{v_{0}}\left(\alpha^{p}\right)$ and $F_{v_{i}}\left(\alpha^{p \prime}\right)$ be the sets of flags at these boundaries. Add new vertices to all the flags and identify two vertices if two flags make up an angle with angle marking 0 . Now each of the sets of vertices obtained from $F_{v_{0}}\left(\alpha^{p}\right)$ and $F_{v_{i}}\left(\alpha^{p^{\prime}}\right)$ comes in a linear order by which we enumerate them, where we actually enumerate the new vertices obtained from $F_{v_{0}}\left(\alpha^{p}\right)$ in the inverse order, that is, last to first. Now we identify all the vertices with the same number from the enumeration. We call the flags of $F_{v_{0}}\left(\alpha^{p}\right)$ and $F_{v_{i}}\left(\alpha^{p^{\prime}}\right)$ flags from different sides, since they lie on opposite sides of the separating curve that is the image of the two glued boundaries. Notice that since we are in the perfectly matched case, there are equally many vertices, and if such a vertex has more than two flags, only one side has more than two flags. If there are only two flags $\left\{f_{1}, f_{2}\right\}$ at a vertex, we delete the vertex and glue the edges by removing the two flags $\left\{f_{1}, f_{2}\right\}$ and setting $l\left(l_{\alpha} p\left(f_{1}\right)\right):=l_{\alpha^{\prime}}\left(f_{2}\right)$. In the case that there are more flags, say $f$ on one side and $\left(f_{1}, \ldots, f_{l}\right)$ on the other side enumerated in their linear order, we duplicate $(l-1)$-times the flag $f$ and glue the $l$ copies to the $f_{i}$ in the obvious linear order. In this way, we obtain new angles, namely the angles between the various copies of $f$. We mark all these angles by 0 . We furthermore forget all the angle markings at $v_{i}$ and $v_{0}$ and retain all other markings.

Again, there are two more steps:

(1) if the arc graphs are twisted at the respective boundaries, we set the composition zero;

(2) if the gluing results in closed loops, that is, embedded arcs that do not touch any boundary as edges, set the contribution zero.

As above we call this gluing the topological gluing and call the gluing which omits step (1) the algebraic gluing.

Self-gluing. The conditions of two boundaries being perfectly matched translate in a straightforward fashion to the case of two boundaries of the same partitioned arc graphs. We define the self-gluing by the same procedure.

An example of such a gluing is given in Figure 3.

Remark 1.19. The second condition is needed in order to stay inside the current framework. It is interesting to observe what these closed loops correspond to in different settings. In the geometric setup they can be understood in terms of general 

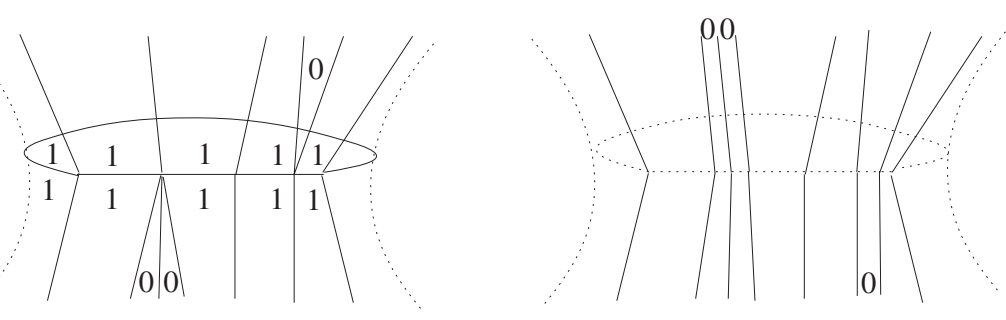

Figure 3. An example of a gluing in the perfectly matched case.

foliations; see, e.g., [KP]. In the Hochschild setting, see $\$ 3.2 .2$ that each closed loop contributes a factor that equals the dimension of the algebra as the trace over the Casimir element.

The first and second conditions are natural from the topological point of view and are necessary if we wish to have an operadic map from $\overline{\mathscr{E}}^{e}$ to $\mathcal{P} \overline{\mathscr{E}}^{e}$. See $\S 5$ for more comments.

Proposition 1.20. The gluings defined above turn $\mathcal{P} \angle \overline{\mathscr{E}}$ bi-graded by the number of boundaries minus one (as the operadic degree) and the genus (as the genus degree) into an partial modular operad.

Moreover, this partial modular operad structure augmented by setting zero any thus far not defined gluing is an operad structure when restricted to $\mathcal{P} \angle \overline{\mathcal{E}}^{e}$, and a di-operad structure on $\mathcal{P}^{<} \overline{\mathrm{Arc}}^{i \leftrightarrow o}$ if one restricts the gluings to gluing only inputs to outputs. Finally, using consecutive self-gluings on $\mathcal{P} \angle \overline{\mathcal{A r c}}^{i \leftrightarrow o}$ to glue all "ins" to all "outs" of collections of arc graphs, the partial modular operad structure induces a PROP structure.

Also, similarly, there is a PROP structure - which we call the algebraic PROP

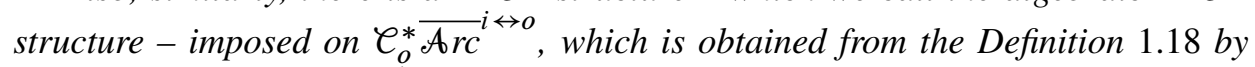
omitting the final step (1). ${ }^{4}$

Proof. The equivariance with respect to the symmetric group actions is immediate. The associativity of the partial operations is also straightforward. Now in both of the special cases the condition of perfect matching is built in and does not change under gluing. In the first case perfect matching reduces to matching, since all the angles are marked by 1 . In the case of $\mathcal{P} \angle \overline{\mathcal{A} r c} i \leftrightarrow o$ all the boundaries which are to be glued are also perfectly matched if they are angle matched since the In boundaries are again all marked by 1 . This condition does not change under gluing, so the result follows by a straightforward calculation.

\footnotetext{
${ }^{4}$ Notice that the situation of step (2) actually never occurs.
} 
Remark 1.21. We only defined the gluing in the non-degenerate case. The general case can be treated in several ways. One is to use the shuffle combinatorics, like in the definition of gluing for $\mathcal{A} r c$ [KLP], [K5]. Here one can either average over the occurring combinatorial types, or not. Another possibility is to go outside the present framework and allow arcs running to punctures, in which case one simply identifies the vertices and if their valence is more than two one leaves them in the surface as new punctures. This is reminiscent of the procedure for open gluing in [KP]. We shall not need these considerations in the following, but an extension of the gluing is of general interest and deserves further study.

Lemma 1.22. Let $\alpha, \beta \in \overline{\operatorname{Arc}}^{i \leftrightarrow o}$. Then

$$
\mathscr{P}^{L}(\alpha) \circ_{i} \mathcal{P}^{L}(\beta)= \pm \mathcal{P}^{L}\left(\alpha \circ_{i} \beta\right),
$$

and this map is $\$_{n}$-equivariant. Thus $\mathcal{P}^{<}$is an operadic map if we use the operadic composition in $\operatorname{Gr} \mathcal{C}_{o}^{*}\left(\overline{\mathcal{A r C}}^{i \leftrightarrow o}\right) \cong C C_{*}\left(\overline{\mathcal{A r C}}_{1}^{i \leftrightarrow o}\right)$ on the right-hand side.

Proof. Since in the standard marking all the angles on the inputs are labelled by one, while the outputs are labelled by zero, we have the following cases. Two partitioning angles are glued. This does not change the number of non-partitioning angles. An input non-partitioning angle is glued to an output partitioning angle. In this case the new edges form a non-partitioning angle. Finally, an output non-partitioning angle is glued to a doubled incoming edge, which again results in a non-partitioning angle. So we find that the number of non-partitioning angles is additive. (See Figure 4 for an example.) This means that only the graphs of top degree appear. On the other hand it is clear that all possible partitioned graphs of $\alpha \circ \beta$ with the maximal number of non-partitioning angles appear, which means that we obtain exactly the graphs of $\mathcal{P}^{\angle}\left(\alpha \circ_{i} \beta\right)$.

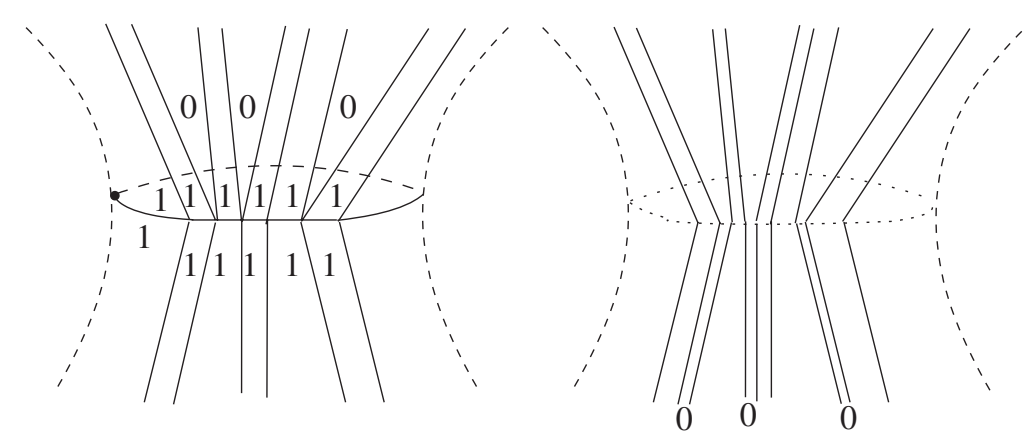

Figure 4. An example of the gluing in $\mathcal{P} \angle \overline{\mathcal{A} r c} i \leftrightarrow o$. 


\subsection{Graded version for angle marked graphs}

Proposition 1.23. $\mathcal{P} \angle$ is an operadic map - under the embedding given by marking all angles by one - between $\mathrm{Gr} \mathcal{A} r c_{\#}^{0}$ and its image $\mathrm{Gr} \mathcal{P}<\mathcal{A} r c_{\#}^{0}$ in $\mathrm{Gr} \mathcal{P} \overrightarrow{\mathcal{E}}$. In other words, let $\alpha, \beta \in \mathrm{Gr} \mathcal{A} r c_{\#}^{0}$, then equation (1.6) also holds true if we define the left-hand operation to lie inside $\mathrm{Gr} \mathcal{P} \overline{\mathscr{E}}$.

Proof. Notice that for this embedding of the angle marked graphs there are no restrictions for the partial gluings. In both cases the boundaries are perfectly angle matched as soon as they are angle matched. In the graded partitioned gluing, we retain the summands that correspond to the highest possible number of non-partitioning angles. These in turn correspond to the angles of the non-partitioned graphs, and thus the conditions imposed on the gluing correspond to each other under the map $\mathcal{P}^{L}$.

\section{Operadic correlation functions}

In this section, we introduce operadic correlation functions, which can be thought of as the generalization of an algebra over a cyclic operad to the dg-setting. In order to get to the main definition, we first set up some notation.

Given a pair $(A, C)$ with $A$ a vector space and $C=\sum c^{(1)} \otimes c^{(2)} \in A \otimes A$, we define the following operations:

$$
\circ_{i}: \operatorname{Hom}\left(A^{\otimes n+1}, k\right) \otimes \operatorname{Hom}\left(A^{\otimes m+1}, k\right) \rightarrow \operatorname{Hom}\left(A^{\otimes n+m}, k\right),
$$

where for $\phi \in \operatorname{Hom}\left(A^{\otimes n+1}, k\right)$ and $\psi \in \operatorname{Hom}\left(A^{\otimes m+1}, k\right)$

$$
\begin{gathered}
\phi \circ_{i} \psi\left(a_{1} \otimes \cdots \otimes a_{n+m}\right) \\
=\sum \phi\left(a_{1} \otimes \cdots \otimes a_{i-1} \otimes c^{(1)} \otimes a_{i+m} \otimes \cdots \otimes a_{m+n}\right) \\
\cdot \psi\left(c^{(2)} \otimes a_{i} \otimes \cdots \otimes a_{i+m-1}\right) .
\end{gathered}
$$

Definition 2.1. A set of operadic correlation function for a cyclic linear operad $\mathcal{O}$ is a tuple $\left(A, C,\left\{Y_{n}\right\}\right)$ where $A$ is a vector space, $C=\sum c^{(1)} \otimes c^{(2)} \in A \otimes A$ is a fixed element and $Y_{n+1}: \mathcal{O}(n) \rightarrow \operatorname{Hom}\left(A^{\otimes n+1}, k\right)$ is a set of multilinear maps. The maps $\left\{Y_{n}\right\}$ should be $\mathbb{S}_{n+1}$-equivariant, and for $o p_{n} \in \mathcal{O}(n), o p_{m} \in \mathcal{O}(m)$ we have

$$
Y_{n+m}\left(o p_{n} \circ_{i} o p_{m}\right)=Y_{n+1}\left(o p_{n}\right) \circ_{i} Y_{m+1}\left(o p_{m}\right),
$$

where the $\circ_{i}$ on the left is the multiplication of equation (2.1) for the pair $(A, C)$.

We call the data $\left(A,\left\{Y_{n}\right\}\right)$ of an algebra and the $\mathbb{S}_{n+1}$-equivariant maps correlation functions or simply correlators for $\mathcal{O}$. 
2.1. Correlators for algebras over cyclic operads. An example is given by an algebra over a cyclic operad. Recall that this is a triple $\left(A,\langle\rangle,,\left\{\rho_{n}\right\}\right)$ where $A$ is a vector space, $\langle$,$\rangle is a non-degenerate bilinear pairing and \rho_{n}: \mathcal{O}(n) \rightarrow \operatorname{Hom}\left(A^{\otimes n}, A\right)$ are multilinear maps, also called correlators, that satisfy:

i) $\rho\left(o p_{n} \circ_{i} o p_{m}\right)=\rho\left(o p_{n}\right) \circ_{i} \rho\left(o p_{m}\right)$ where $\circ_{i}$ is the substitution in the $i$-th variable;

ii) the induced maps $Y_{n+1}: \mathcal{O}(n) \rightarrow \operatorname{Hom}\left(A^{\otimes n+1}, k\right)$ given by

$$
Y_{n+1}\left(o p_{n}\right)\left(a_{0} \otimes \cdots \otimes a_{n}\right):=\left\langle a_{0}, \rho\left(o p_{n}\right)\left(a_{1} \otimes \cdots \otimes a_{n}\right)\right\rangle
$$

are $\$_{n+1}$-equivariant.

Notation 2.2. Given a finite dimensional vector space $A$ with a non-degenerate pairing $\langle\rangle=,\eta \in \check{A} \otimes \check{A}$, let $C \in A \otimes A$ be dual to $\eta$ under the isomorphism induced by the pairing and call it the Casimir element. It has the following explicit expression: Let $e_{i}$ be a basis of $V$, let $\eta_{i j}:=\left\langle e_{i}, e_{j}\right\rangle$ be the matrix of the metric and let $\eta^{i j}$ be the inverse matrix. Then $C=\sum_{i j} e_{i} \eta^{i j} \otimes e_{j}$.

Lemma 2.3. The assignment $\left(A,\langle\rangle,,\left\{\rho_{n}\right\}\right) \mapsto\left(A, C,\left\{Y_{n}\right\}\right)$, where the $Y_{n}$ are defined as in equation (2.4), gives a 1-1 correspondence between the algebra structure over a cyclic operad and operadic correlation functions, which is functorial.

Proof. We have defined the map in one direction. To give the inverse map, we set

$$
\rho_{n}\left(a_{1} \otimes \cdots \otimes a_{n}\right):=\sum Y_{n+1}\left(c^{(1)}, a_{1} \otimes \cdots \otimes a_{n}\right) c^{(2)} .
$$

A direct calculation verifies that these assignments are inverse to each other. The compatibility of the $\mathbb{S}_{n+1}$ operations is manifest. Finally, it is clear that this construction is functorial for maps of cyclic operads and maps of algebras with a non-degenerate pairing.

\subsection{Operadic correlations functions with values in a twisted $\mathscr{H} o m$ operad}

Definition 2.4. Let $\left(A,\langle\rangle,,\left\{Y_{n}\right\}\right)$ be as above, and let $\mathscr{H}=\{\mathscr{H}(n)\}$ with $\mathscr{H}(n) \subset$ $\operatorname{Hom}\left(A^{\otimes n}, A\right)$ as $k$-modules be an operad where the $\mathbb{\$}_{n}$ action is the usual action, but the operad structure is not necessarily the induced operad structure. Furthermore assume that $\rho_{Y_{n+1}} \in \mathscr{H}(n)$. We say that the $\left\{Y_{n}\right\}$ are operadic correlation functions for $\mathcal{O}$ with values in $\mathscr{H}$ if the maps $\rho$ are operadic maps from $\mathcal{O}$ to $\mathscr{H}$. We will also say that we get an action of $\mathcal{O}$ with values in $\mathscr{H}$.

2.2.1. Signs. As in the case of the Deligne conjecture one twist which we have to use is dictated by picking sign rules. In the case of Deligne's conjecture this could be done by mapping to the brace operad Brace (see, e.g., [K4]) or by twisting the 
operad Hom by lines of degree 1 as in $\S 1.3 .4$ (see, e.g., [KS1]). In what follows, our actions will take values on operads that are naturally graded and, moreover, we will identify the grading with the geometric grading by, for instance, the number of edges or the number of angles, etc. The signs will then automatically match up if we use the procedure of $\S 1.3 .4$ at the same time for both the graph side and the $\mathscr{H} o m$ side, i.e., for the operad $\mathscr{H}$. In fact, this approach unifies the two sign conventions mentioned above on the subspace of operations corresponding to $\mathscr{L} \mathcal{T}_{r e e_{c p}}$.

2.3. Correlators for dg algebras. Let $(V, d)$ be a complex whose homology algebra $H:=H(V, d)$ has a non-degenerate pairing $\langle$,$\rangle . Let C$ be the Casimir element of $\langle$,$\rangle . Let Z=\operatorname{ker}(d)$ and let $s$ be a section of the projection $Z \rightarrow H$.

Definition 2.5. If $(V, d)$ is a complex, we call a set of correlation functions for $V$ operadic chain level correlation functions if they are operadic correlation functions for $Z(A)=\operatorname{ker}(d)$.

Proposition 2.6. Let $\mathcal{O}:=\{\mathcal{O}(n)\}$ be a cyclic operad, and let $(V, d,\langle\rangle$,$) and s$ be as above. Let $Z=\operatorname{ker}(d)$ and let $Y_{n+1}: \mathcal{O}(n) \rightarrow \operatorname{Hom}\left(V^{\otimes n+1}, k\right)$ be a collection of $\mathbb{\$}_{n+1}$-equivariant maps whose values only depend on the classes in $H$, that is, if $\left[a_{i}\right]=\left[b_{i}\right] \in H$ where $a \mapsto[a]$ is the projection map $Z \rightarrow H$, then $Y_{n}\left(a_{1} \otimes \cdots \otimes\right.$ $\left.a_{n}\right)=Y_{n}\left(b_{1} \otimes \cdots \otimes b_{n}\right)$. Furthermore, assume that the induced maps $\bar{Y}_{n+1}: \mathcal{O}(n) \rightarrow$ $\operatorname{Hom}\left(H^{\otimes n+1}, k\right)$ are operadic correlation functions for the cyclic operad. Then, for any section $s$ of the projection map $Z \rightarrow H$, the collection $\left\{Y_{n}\right\}$ is a set of operadic correlation functions for $(Z,(s \otimes s)(C))$.

Proof. Straightforward.

Definition 2.7. A quasi-Frobenius algebra is a triple $(A, d,\langle\rangle$,$) where (A, d)$ is a unital dg algebra whose homology algebra $H:=H(A, d)$ is finite dimensional, and has a non-degenerate pairing $\langle$,$\rangle , and is a Frobenius algebra for this pairing. A quasi-$ Frobenius algebra with an integral is a triple $\left(A, d, \int\right)$ where $\int: A \rightarrow k$ is a linear map such that:

i) For all $a \in A: \int d a=0$.

ii) $(A, d,\langle\rangle$,$) is a quasi-Frobenius algebra, where \langle a, b\rangle:=\int a b$. The co-cycles of a quasi-Frobenius algebra with an integral are the subalgebra $Z=\operatorname{ker}(d) \subset A$ of the algebra above.

A natural example of a quasi-Frobenius algebra with an integral is $A=C^{*}(M)$, the co-chains of a compact manifold $M$. 
Example 2.8. Let $\mathcal{C y c}_{\mathrm{c}}:=$ Assoc [1] be the cyclic operad obtained by shifting the associative operad $\mathcal{A s s o c}$ by 1 , that is, $\mathcal{C} y c(n)$ is the permutation representation $\mathbb{\$}_{n+1}$ on $\{0, \ldots, n\}$ with $\mathbb{S}_{n}$ acting on $\{1, \ldots, n\}$.

Let $(A, d)$ be an associative dg algebra with an integral, i.e., a function $\int: A \rightarrow k$ which satisfies $\int a b=(-1)^{\operatorname{deg}(a) \operatorname{deg}(b)} \int b a$. Set $\langle a, b\rangle=\int a b$ and suppose that $\langle$,$\rangle is non-degenerate on H=H(A, d)$.

For $\sigma_{n} \in \mathbb{S}_{n}$ viewed as a generator of $\mathcal{C}_{y c}(n-1)$ we define

$$
Y_{n+1}\left(\sigma_{n}\right)\left(a_{0} \otimes \cdots \otimes a_{n-1}\right):= \pm \int a_{\sigma(0)} \cdots a_{\sigma(n-1)},
$$

where \pm is the sign of the permutation of the elements $a_{i}$. Then by the proposition above this is a set of operadic correlation functions.

Fixing a section $s$ of the map $Z \rightarrow H, a \mapsto[a]$ the operations

$$
\rho_{n}\left(\operatorname{id}_{n+1}\right)\left(a_{1} \otimes \cdots \otimes a_{n}\right)= \pm s\left(\left[a_{1}\right] \ldots\left[a_{n}\right]\right)
$$

yield operadic correlation functions.

In the case that $A=C^{*}(M)$ we see that we recover the cup product up to homotopy. This is enough to characterize the cup product of two closed co-chains inside any integral.

2.4. Example: The tensor algebra. As a simpler example than the Hochschild cochains, we will consider the action of the various algebraic structures on a tensor algebra. This type of action is interesting in its own right. Moreover it is related to the actions on the simplicial co-chain complexes regarded in [MS3].

Let $V$ be a vector space and let $T V=\bigoplus_{n \geq 0} V^{\otimes n}$ be its tensor algebra. Let $\overline{T V}=\bigoplus_{n \geq 1} V^{\otimes n}$ be the reduced tensor algebra.

Fix a non-degenerate symmetric pairing $\langle$,$\rangle on V$. This pairing induces a symmetric non-degenerate pairing on $T V$, so that we can consider $T V$ (and $\overline{T V}$ ) as a candidate of an algebra over a cyclic operad.

2.4.1. Correlators. Suppose that $\alpha \in\left(\overline{\mathscr{E}}^{e}\right)^{k}(n)$ and $p \in P(m, k)$. We define an $Y\left(\alpha^{p}\right): \overline{T V}^{\otimes n} \rightarrow k$ as follows. Let $n_{i}:=\#\{$ flags at the boundary $i\}$ of the arc graph of $\alpha^{p}$.

If $v \notin \bigotimes_{i=1}^{k} V^{\otimes n_{i}} \subset \overline{T V}^{\otimes n}$, we set $\alpha^{p}(v)=0$. If $v=\bigotimes_{i=1}^{k}\left(v_{i 1} \otimes \cdots \otimes v_{i n_{i}}\right)$ then we associate to each of the flags $f$ of the arc graph of $\alpha^{p}$ incident to the boundary $i$ the element $v_{f}:=v_{i,|f|}$, where again $|f|$ is the position of the flag in its linear order at the vertex $i$. Let $E$ be the set of edges of the arc graph of $\alpha^{p}$. Then we define

$$
Y\left(\alpha^{p}\right)(v):=\prod_{e=\{f, l(f)\} \in E}\left\langle v_{f}, v_{l(f)}\right\rangle
$$


and extend to a multilinear map. It is straightforward to check that this defines operadic correlation functions for $\mathcal{P} \overline{\mathscr{E}}^{e}$ with the algebraic gluings in the case that the final step (2) of $\S 1.4 .1$ is not applicable.

We extend to operations of $\mathcal{P} \overline{\mathscr{E}}$ and hence $\overline{\mathscr{E}}$ on $T V$ by using the isomorphisms $V \otimes_{k} k \simeq V$ to "decorate" the empty boundaries with copies of $k$. This gives well-defined multilinear maps.

\subsubsection{Actions on the tensor algebra of a vector space with values in a twisted Hom operad}

Definition 2.9. We let $\mathcal{C} \mathcal{M}(n) \subset \operatorname{Hom}(T V)(n)$ be the image of all the operations of $\mathcal{P} \overline{\mathscr{G}}(n)$ by considering 0 as "out".

Notation 2.10. We will use the following notation: $\Delta^{l}: T V \rightarrow T V^{\otimes l+1}$ is the iteration of $\Delta$ given by $\left(\Delta \otimes \mathrm{id}^{\otimes l}\right) \circ\left(\Delta \otimes \mathrm{id}^{\otimes l-1}\right) \circ \ldots \circ(\Delta \otimes \mathrm{id}) \circ \Delta$.

We write $\eta^{m}$ for the map $V^{\otimes 2 m} \rightarrow k, \eta\left(a_{1} \otimes \cdots \otimes a_{2 m}\right)=\prod_{i=1}^{m} \eta\left(a_{i}, a_{2 m-i}\right)$.

Proposition 2.11. After dualizing to obtain elements in $\operatorname{Hom}\left(T V^{\otimes n+1}, k\right)$ any element in $\mathcal{C}_{\mathcal{M}}(n)$ can be written uniquely as

$$
\bigotimes_{j=1}^{\frac{1}{2} \sum_{i}\left(n_{i}+1\right)} \eta^{m_{j}} \circ \sigma \circ \bigotimes_{i=0}^{n} \Delta^{n_{i}}
$$

where $\sigma$ is a permutation of the $\sum_{i}\left(n_{i}+1\right)$-factors of TV. Set $l=\frac{1}{2} \sum_{i}\left(n_{i}+1\right)-1$. Then $\mathcal{C}_{\mathcal{M}}$ is graded by $l$. Moreover, the composition in $\mathcal{C}_{\mathcal{M}}$ respects the induced filtration of elements of degree $\leq l$. Finally, the decomposition identifies $\mathcal{C}_{\mathcal{M}}$ with the subspace of $\operatorname{Hom}(T V)$ obtained by dualization for the subspace generated by the coproduct, permutations and $\eta$ in $\bigoplus_{n} \operatorname{Hom}\left(T V^{\otimes n+1}, k\right)$. That is, we obtain correlation functions with values in $\mathcal{C} \mathcal{M}$.

Proof. The first statement is clear by the definition of $\mathcal{C} \mathcal{M}$ as the image. The last statement is also straightforward by arranging the operation in the specified order. On the other hand it is easy to give the arc graph in $\mathcal{P} \overline{\mathscr{E}}(n)$ by drawing one arc for each factor of $\eta$ with the incidence relations given by $\sigma$. This identifies the two subspaces. In this identification there is one factor of $\Delta$ for each non-partitioning inner angle. The last claim that the operations respect the filtrations is clear after identifying $k$ with the dimension of the cell, that is, the number of edges minus one, of the underlying graph for the operation. The mentioned equality follows from the combinatorial identity $\left|\angle_{\text {inner }}\right|+\mid \angle$ outer $|=|$ Flags $|=2|$ edges $\mid$.

Proposition 2.12. For any vector space $V$ with a non-degenerate pairing, TV is an algebra over the algebraic PROP $\mathcal{P A} r c^{i \leftrightarrow i}$. Moreover this action passes to the associated graded and gives an action of $C C_{*}(\mathcal{A})$ with values in $\mathrm{Gr} \mathcal{C} \mathcal{M}$. 
Proof. We extended the definition of correlators above to correlators for $\bar{\varepsilon}$ and hence to $C C_{*}(\mathcal{A})$ on $T V$ using the isomorphism $V \otimes_{k} k \cong V$. We use the Casimir element to dualize, and thus we only have to show that the resulting structure is that of an algebra over a cyclic operad. The $\mathbb{\$}_{n+1}$ equivariance is manifest. After dualizing, the gluing on the flags in the operadic composition turns into the identity map id: $A \rightarrow A$ so that indeed the gluing ${ }_{i}$ on $\mathcal{P A} r c^{i}{ }^{i t i}$ maps to insertion at the $i$-th place in $\operatorname{Hom}(T V)$. Dealing with the extra steps (1) and (2), we see that on the side of Hom(TV) they would not yield zero. In $\mathcal{P A} r c^{i \leftrightarrow i}$ closed loops cannot appear, hence step (2) is avoided. Using the algebraic gluing, we get agreement for the two operations. In the graded case, the contributions of (1) and (2) are projected away on the side of $\mathcal{C}_{\mathcal{M}}$, as is the case in $C C_{*}(\mathcal{A})$ where these contributions come from lower degree cells which are again projected out. Moreover as mentioned above the grading in both cases is by the number of arcs of the underlying graphs -1 .

Remark 2.13. Without the pairing, we could still define operations for graphs of $\operatorname{Gr} \mathcal{C}_{o}^{*}\left(\overline{\mathcal{A C r C}}^{i \leftrightarrow o}\right) \simeq C C_{*}\left(\overline{\mathcal{A C r}}_{1}^{i \leftrightarrow o}\right)$.

Remark 2.14. The condition of genus zero also has a nice algebraic counterpart in this setting, and that is the condition that the permutation is only a shuffle.

Example 2.15. An example where the grading is not respected occurs when one considers $\Delta \circ \mu_{\otimes}: T V \otimes T V \rightarrow T V \otimes T V$. The generic number of shuffles will be 1 , but there will be a summand corresponding to id $\otimes$ id which requires no shuffle.

This example is very instructive, since it is this sort of behavior that is very characteristic for our actions and their associated graded ones.

We can decompose

$$
\Delta \circ \mu_{\otimes}=\left(\mathrm{id} \otimes \mu_{\otimes}\right) \circ(\Delta \otimes \mathrm{id})+\mathrm{id} \otimes \mathrm{id}+\left(\mu_{\otimes} \otimes \mathrm{id}\right) \circ(\mathrm{id} \otimes \Delta) .
$$

As is well known, this means that $T V$ as an algebra and coalgebra is not Frobenius but rather has the operations usually forming the other side of the Frobenius equation as summands of the operation $\Delta \circ \mu_{\otimes}$; plus there is one more summand of lower degree, namely id $\otimes$ id. The associated graded will project out this term.

Extending to cycles, we obtain:

Theorem 2.16. For a complex $(V, d)$ over $k$ with a pairing $\langle$,$\rangle that satisfies$

i) $\langle d v, w\rangle=-\langle w, d v\rangle$ for all $v, w \in V$, and

ii) the induced pairing on $H=H(V, d)$ is non-degenerate,

the formulas of $\$ 2.4$ extended as in Proposition 2.12 yield correlation functions on the tensor algebra $T V$ which are operadic on $T Z$, that is, operadic chain level correlation functions. 
2.5. Polygon correlation functions. Other examples of operadic correlation functions come from operads of polygons. It is this type of example which we generalize to obtain the correlators for $\mathcal{P} A r c^{L}$.

Let $p_{n}$ be the regular $n$-gon and denote its sides by sides $\left(p_{n}\right)$. We let $\mathcal{P o l y}(n)=$ the free Abelian group generated by $\left\{\right.$ Lab: $\left.\operatorname{sides}\left(p_{n}\right) \rightarrow\{0, \ldots, n-1\}\right\}$. Similarly, let $P_{2 n}$ be the regular $2 n$-gon with a fixed choice of a preferred set of $n$ non-intersecting sides, which we call Sides $\left(P_{2 n}\right)$. Then $\operatorname{Poly}_{2}(n)$ is the free Abelian group generated by $\left\{\operatorname{Lab}: \operatorname{Sides}\left(P_{2 n}\right) \rightarrow\{0, \ldots, n-1\}\right\}$. The operad structure $\circ_{i}$ on $\operatorname{Poly}(n)$ is given by gluing the polygons along the sides marked by 0 and $i$, respectively, and deleting the image of the glued side, which is diagonal in the glued object. In $\mathcal{P o l y}_{2}(n)$ we also merge the two pairs of non-labelled sides on the two sides of the deleted diagonal. The $\mathbb{S}_{n+1}$ action is given by permuting the labels on the labelled sides.

Remark 2.17. These operads are different incarnations of the operad $\mathcal{C}_{y c}$. The map from $\mathcal{P o l y}$ to trees is just given by marking the center of the polygon and the middle of the sides by a vertex, connecting the vertices of the sides to the center vertex, and carrying over the labelling. This yields an isomorphism to the usual pictorial way of defining $\ell_{y c}$ in terms of planar corollas (see, e.g., [K1]). An isomorphism from $\mathcal{P} o l y$ to $\mathcal{P o l y}_{2}$ is given by blowing up the vertices of $p_{n}$ to sides and choosing the original sides to be the preferred set of $n$ sides of $P_{2 n}$. The inverse is given by contracting the non-preferred sides.

Let $\left(A, d, \int\right)$ be a quasi-Frobenius algebra with an integral. We define correlation functions as

$$
\begin{aligned}
Y(\mathrm{Lab}: & \left.\left.\operatorname{sides}\left(p_{n}\right)\right) \rightarrow\{0, \ldots, n-1\}\right)\left(a_{\mathrm{Lab}^{-1}(0)} \otimes \cdots \otimes a_{\mathrm{Lab}^{-1}(n-1)}\right) \\
= & \int a_{\mathrm{Lab}^{-1}(0)} \cdots a_{\mathrm{Lab}^{-1}(n-1)}, \\
Y(\mathrm{Lab} & \left.: \operatorname{Sides}\left(P_{2 n}\right)\right)\left(a_{\mathrm{Lab}^{-1}(0)} \otimes \cdots \otimes a_{\mathrm{Lab}^{-1}(n-1)}\right) \\
= & \int a_{\mathrm{Lab}^{-1}(0)} \cdots a_{\mathrm{Lab}^{-1}(n-1)} .
\end{aligned}
$$

Here and in the following we will frequently drop the subscripts on the $Y_{n}$ since they can be deduced from the expression.

Lemma 2.18. Given a quasi-Frobenius algebra with an integral $\left(A, d, \int\right)$, the equations above define operadic correlation functions for Poly and $\mathcal{P o l y}_{2}$.

Proof. This follows either directly by Proposition 2.6 or by Remark 2.17 and the Example 2.8. 


\section{Correlators for $\mathcal{P}^{L} \mathcal{A}$ on the Hochschild co-chains of a Frobenius algebra}

The natural operations on the tensor algebra $T A$ of an algebra $A$ are the ones generated by the multiplication and co-multiplication $\mu_{\otimes}$ and $\Delta$ of $T A$ as well as the permutations and the multiplication $\mu_{A}: A \otimes A \rightarrow A$. In order to incorporate the later operations into the picture, we will have to modify the correlators of $\$ 2.4$ a little and introduce operations which act "internally", that is, operations which are associated to complementary regions or dually in the case of $\mathcal{A} r c_{\#}^{0}$ at the vertices of the dual graph.

\subsection{Graph correlation functions also known as Feynman rules}

Example 3.1. We will recall how to define correlation functions for ribbon graphs (see, e.g., [Ko3], [KM]) by using so-called Feynman rules. Although our action will be slightly different, the underlying principle is similar, and this easier example will be instructive.

Let $\Gamma$ be a ribbon graph with vertices of valence at least 3 . Let $A$ be an algebra with a non-degenerate pairing, which gives an isomorphism of $A$ with its dual $\mathscr{A}$. Let $C$ be the Casimir element. Let $\phi: V_{\Gamma} \rightarrow \mathscr{H o m}(A, A)$ be a map which

i) preserves degree, i.e., $\phi(v) \in \mathscr{H o m}(A, A)(\operatorname{val}(v-1)) \simeq \operatorname{Hom}\left(A^{\otimes \operatorname{val}(v)}, k\right)$;

ii) has a cyclic image, i.e.,

$$
\phi(v)\left(a_{0} \otimes \cdots \otimes a_{n}\right)= \pm \phi(v)\left(a_{1} \otimes \cdots \otimes a_{n} \otimes a_{0}\right) \quad \text { for all } v f \in V_{\Gamma},
$$

where \pm is the permutation super-sign.

Set

$$
Y(\Gamma)(\phi):=\left(\bigotimes_{v \in V_{\Gamma}(\tau)} \phi(v)\right)\left(\bigotimes_{e \in E_{\Gamma}} C\right) \in k .
$$

Remark 3.2. Let $\mathcal{G}$ be a class of graphs, e.g., the set of all planar trees or ribbon graphs. Functions of the type $\phi: \mathscr{G} \rightarrow \mathscr{H o m}(A, A)$ are sometimes called Feynman rules if the map $\phi$ is expressible in local data of the graph, that is, in terms of the flags at each vertex and the edges.

Graphs which have external vertices, that is, vertices with only one adjacent flag, are treated in one of the following ways. One can either partition the external vertices into a set In and a set Out, say of cardinalities $p$ and $q$, and view $Y(\Gamma)^{p, q} \in \operatorname{Hom}\left(A^{\otimes p}, A^{\otimes q}\right)$, or view all vertices as inputs $Y(\Gamma) \in \operatorname{Hom}\left(A^{\otimes p+q}, k\right)$. An example is given by associating elements of $A$ to the flags of the external vertices of a ribbon graph with tails and plugging in copies of $C$ into the operations by decorating the internal edges and then contracting like in (3.1) 
Example 3.3. An important example for Feynman rules $\phi$ for ribbon graphs $\Gamma$ is given by using an $A_{\infty}$-algebra [Ko3]. Here $\phi(v)=\phi(\operatorname{val}(v))=\mu_{n}$, where now $\mu_{n}: A^{\otimes n} \rightarrow A$ is one of the structure maps of the $A_{\infty}$-algebra.

Example 3.4. In [K2] we also used Feynman rules for trees with external vertices. They are related to the operations that we describe below, by moving to the dual graph and then to the intersection graph. They are hence decidedly different for the Example 3.3 above, which directly deals with ribbon graphs.

3.2. $A \boldsymbol{A c}^{\perp}$ correlation functions. The idea of how to obtain the correlation functions for the tensor algebra is very nice in the $\mathcal{A} r c$ picture where it is based on the polygon picture. This polygon picture can be thought of as an IRF (interaction round a face) picture for a grid on a surface which is dual to the ribbon picture. For this we would modify the arc graph by moving the arcs a little bit apart as described in $\S 1.3 .1$. Then the complementary regions of partitioned quasi-filling arc graphs $\mathcal{P} \overline{\mathscr{E}}_{\#}$ are $2 k$-gons whose sides alternatingly correspond to arcs and pieces of the boundary. The pieces of the boundary correspond to the angles of the graph, and of course any polygonal region corresponds to a cycle of the arc graph. If the graph $\alpha^{p}$ has an angle marking, then the sides of the polygons corresponding to the boundaries will also be marked. We fix the following notation. For an angle marked partitioned arc graph $\alpha^{p} \in \mathcal{P}<\overline{\mathscr{G}}_{\#}$ let Poly $\left(\alpha^{p}\right)$ be the set of polygons given by the complementary regions of $\alpha^{p}$ when treated as above. For $\pi \in \operatorname{Poly}\left(\alpha^{p}\right)$, let Sides' be the sides corresponding to the angles which are marked by 1 and $\operatorname{Sides}^{\prime}\left(\alpha^{p}\right)$ be the union of all of these sides. If we denote $L^{+}(\Gamma)=\left(m k^{L}\right)^{-1}(1)$ there is a natural bijection between $L^{+}\left(\alpha^{p}\right)$ and $\operatorname{Sides}^{\prime}\left(\alpha^{p}\right)$.

3.2.1. Correlation functions on the tensor algebra of an algebra. Fix an algebra $A$ with a cyclic trace, i.e., a map $\int: A \rightarrow k$ which satisfies $\int a_{1} \ldots a_{n}=$ $\pm \int a_{n} a_{1} \ldots a_{n-1}$ where \pm is the standard sign.

Now for $\pi \in \operatorname{Poly}\left(\alpha^{p}\right)$ set

$$
Y(\pi)\left(\bigotimes_{s \in \operatorname{Sides}^{\prime}(\pi)} a_{s}\right)=\int \prod_{s \in \operatorname{Sides}^{\prime}(\pi)} a_{s} .
$$

Notice that we only have a cyclic order for the sides of the polygon, but $\int$ is (super)invariant under cyclic permutations, so that if we think of the tensor product and the product as indexed by sets (3.2) it is well defined.

For an angle marked partitioned arc family $\alpha^{p}$ set

$$
Y\left(\alpha^{p}\right)\left(\bigotimes_{s \in(m k<)^{-1}(1)} a_{s}\right)=\bigotimes_{\pi \in \operatorname{Poly}\left(\alpha^{p}\right)} Y(\pi)\left(\bigotimes_{s \in \operatorname{Sides}^{\prime}(\pi)} a_{s}\right),
$$


where we used the identification of the set $\operatorname{Sides}^{\prime}\left(\alpha^{p}\right)=\amalg_{\pi \in \operatorname{Poly}\left(\alpha^{p}\right)} \operatorname{Sides}^{\prime}(\pi)$ with $\angle^{+}\left(\alpha^{p}\right)$. Since for each $\alpha^{p} \in \mathcal{P}^{\prime} \bar{g}^{e}(n)$ the set of all flags has a linear order, we can think of $Y\left(\alpha^{p}\right)$ as a map $A^{\otimes\left|F\left(\alpha^{p}\right)\right|}=\bigotimes_{i=1}^{n} A^{\otimes\left|F\left(v_{i}\right)\right|} \rightarrow k$, and furthermore as a map to $T A^{\otimes n} \rightarrow k$ by letting it be equal to equation (3.3) as a map from $\bigotimes_{i=1}^{n} A^{\otimes\left|F\left(v_{i}\right)\right|} \subset$ $T A^{\otimes n}$ and setting it to zero outside of this subspace.

Extending linearly, for an angle marked arc family $\alpha \in \mathcal{A} r c^{<}$, we finally define

$$
Y(\alpha):=Y(\mathcal{P}(\alpha))
$$

3.2.2. Correlators for the Hochschild co-chains of a Frobenius algebra. Let $A$ be an algebra and let $\mathrm{CH}^{n}(A, A)=\operatorname{Hom}\left(A^{\otimes n}, A\right)$ be the Hochschild cochain complex of $A$. Set $C_{\text {cyc-bar }}^{n}(A, k)=\operatorname{Hom}\left(A^{\otimes n+1}, k\right) \simeq \operatorname{Hom}\left(C_{n}(A), k\right)$ where $C_{*}(A)$ is the cyclic bar complex. If we endow $C_{\text {cyc-bar }}^{*}(A)$ with the induced differential, then one has a canonical isomorphism $C_{\text {cyc-bar }}^{*}(A) \cong \mathrm{CH}^{*}(A, \check{A})$ as complexes (see, e.g., [L], 1.5.5). And this denoting the Hochschild cohomology by $\mathrm{HH}^{*}(A, A)$ and the cohomology of the complex $C_{\text {cyc-bar }}^{*}$ by $H_{\text {cyc-bar }}^{*}$, we obtain an isomorphism $\mathrm{HH}^{*}(A, A) \simeq H_{\text {cyc-bar }}^{*}(A)$.

Lemma 3.5. For any Frobenius algebra $(A,\langle\rangle$,$) , we have canonical isomorphisms$ $C_{\text {cyc-bar }}^{*}(A) \cong \mathrm{CH}^{*}(A, \check{A}) \cong \mathrm{CH}^{*}(A, A)$ and $H_{\text {cyc-bar }}^{*}(A) \cong \operatorname{HH}^{*}(A, A) \cong \mathrm{HH}^{*}(A, \check{A})$ induced by the isomorphism of $A$ and $\breve{A}$ which is defined by the non-degenerate pairing of $A$.

Proof. The only statement to prove is the last isomorphism. As mentioned, the map on the chain level is induced by the isomorphism of $A$ and $\breve{A}$ defined by the nondegenerate pairing of $A$. The fact that the complexes are isomorphic follows from the well-known fact that the invariance of the pairing $\langle a b, c\rangle=\langle a, b c\rangle$ implies that the isomorphism between $A$ and $\check{A}$ is an isomorphism of $A$ bi-modules, where the bi-module structure of functions $f \in \check{A}$ is given by $a^{\prime} f a^{\prime \prime}(c)=f\left(a^{\prime \prime} c a^{\prime}\right)$; see, e.g., [L].

For any $f \in \mathrm{CH}^{n}(A, A)$ let $\tilde{f} \in \check{A}^{\otimes n}$ be its image under the isomorphism of $\check{A}$, with $A$ defined by the Frobenius structure of $A$.

Given pure tensors $f_{i}=f^{0 i} \otimes f_{1 i} \otimes \cdots \otimes f_{i n_{i}} \in \mathrm{CH}^{n_{i}}(A, A), i \in\{0, \ldots, n\}$, we write $\tilde{f}_{i}=f_{0 i} \otimes \cdots \otimes f_{i n_{i}}$ for their image in $C_{\text {cyc-bar }}^{n_{i}}(A)$. Fix $\alpha \in \operatorname{Arc}^{<}(n)$. Now decorate the sides $s \in \operatorname{Sides}^{\prime}(\alpha):=\left(m k^{\angle}\right)^{-1}(1)$ of the complementary regions, which correspond to pieces of the boundary, by elements of $A$ as follows: for a side $s \in$ Sides $^{\prime}$ let $j$ be its position in its cycle $c_{i}$ counting only the sides of $c_{i}$ in Sides' starting at the side corresponding to the unique outside angle at the boundary given by the cycle. If the number of such sides at the boundary $i$ is $n_{i}+1$, then set $f_{s}:=f_{i j}$. 
Now we set

$$
Y(\alpha)\left(f_{1}, \ldots, f_{n}\right):=Y(\mathcal{P}(\alpha))\left(\bigotimes_{s \in L^{+}\left(\alpha^{p}\right)} f_{s}\right)
$$

We extend this definition by linearity if $f_{i} \in \mathrm{CH}^{n_{i}}(A, A), i \in \bar{n}$. If the condition that $n_{i}+1$ equals the number of Sides' at the boundary $i$ is not met, we set $Y(\alpha)\left(f_{0}, \ldots, f_{n}\right)=0$. An example of a decorated partitioned surface and its polygons is given in Figure 5.
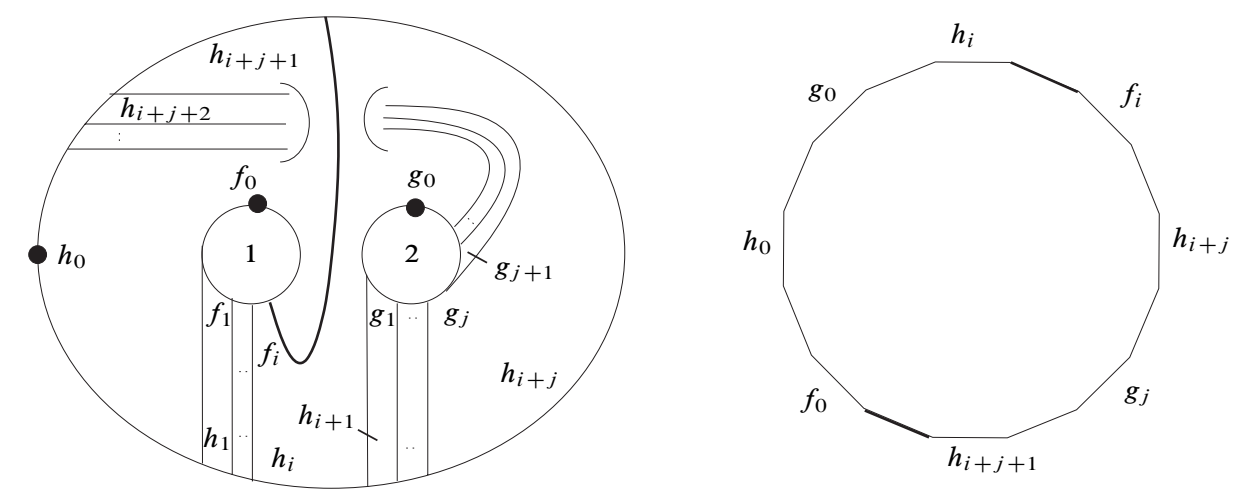

Figure 5. A partitioned arc graph with decorations by elements of $A$ and one of its decorated polygons. The bold line corresponds to the bold edges.

3.3. Ribbon correlation functions. In this section, we give a dual and equivalent picture for the quasi-filling arc graphs in terms of ribbon graphs. Whether in the quasi-filling case one wishes to use ribbon graphs or surfaces is basically a matter of taste. Ribbon graphs seem to be more established, but actually the surfaces seem to be the better geometric fit, especially if one wants to extend the operations "to the boundary" as explained in the next section. Since CFT is, however, usually associated with ribbon graphs rather than arc graphs, we give the details of the construction for this dual picture. We stress, however, that this duality only exists for quasi-filling arc graphs indexing cells of $\mathcal{A} r c_{\#}^{L}$ and that furthermore our correlation functions are completely different from those in [Ko3], where basically a CFT is defined from an $A_{\infty}$-algebra. What we define are morally the correlation functions of the closed string states viewed as deformations of the category of open strings evaluated on a cell of the open moduli space; see $\$ 5$.

3.3.1. Vertex correlation functions. Fix an angle marked ribbon graph $\left(\Gamma, m k^{L}\right)$. Let $v \in V_{\Gamma}$ be a vertex of $\Gamma$. Let $\angle^{+}(v)$ be the subsets of angles of $\angle^{+}(\Gamma)$ whose 
flags are also incident to $v$, and define $Y(v): A^{\otimes L^{+}(v)} \rightarrow k$ by

$$
Y(v)\left(\bigotimes_{\alpha \in L^{+}(v)} a_{\alpha}\right)=\int \prod_{\alpha \in L^{+}(v)} a_{\alpha}
$$

just as for equation (3.2). This is well defined as a function on the tensor product indexed by sets.

3.3.2. Correlators defined by an angle marked ribbon graph. Let $\Gamma^{\prime}$ be a partitioned marked ribbon graph with an angle marking,

$$
Y\left(\Gamma^{\prime}\right)\left(\bigotimes_{\alpha \in L^{+}(\Gamma)} a_{\alpha}\right)=\bigotimes_{v \in V_{\Gamma}} Y(v)\left(\bigotimes_{\beta \in L^{+}(v)} a_{\beta}\right)
$$

Let $\left\{c_{i}\right\}, i=0, \ldots, n-1$ be the set of cycles labelled by $\{0, \ldots, n-1\}$. Let $L^{+}\left(c_{i}\right)$ be the angles corresponding to the flags of the cycle $c_{i}$ which are marked by 1 . Since the ribbon graph is marked, we have an enumeration of all flags and hence all the angles, hence we can think of the equation (3.7) as a map as defined on the subspace $\bigotimes_{i=1}^{n} A^{\left|L^{+}\left(c_{i}\right)\right|} \subset T A^{\otimes n}$ and extend it by zero outside of this subspace.

Finally for an angle marked, marked ribbon graph, we define

$$
Y(\Gamma):=Y(\mathcal{P}(\Gamma))
$$

by extending linearly.

3.3.3. Correlators for the Hochschild complex, the ribbon version. As above, for any $f \in \mathrm{CH}^{n}(A, A)$ let $\tilde{f} \in \check{A}^{\otimes n}$ be its image under the isomorphism of $\check{A}$ and $A$ defined by the Frobenius structure of $A$.

Fix $\Gamma \in \mathcal{R} i b^{\perp}(n)$, and let $c_{i}$ be the cycles of the underlying ribbon graph, also denoted by $\Gamma$. Set $n_{i}=\left|L^{+}\left(c_{i}\right)\right|$. Now for $f_{i} \in \mathrm{CH}^{n_{i}-1}(A, A), i \in\{0, \ldots, n\}$, which are pure tensors, we have $\tilde{f}_{i}=f_{1 i} \otimes \cdots \otimes f_{1 n_{i}}$. Recall that each cycle has a linear order, since the graph is marked. Now decorate the angles of the graph by elements of $A$ as follows: for an angle $\alpha$ let $f$ be the flag of $\alpha$ and let $j$ be its position in its cycle $c_{i}$ starting at the flag preceding the marked flag counting only the elements of $\angle^{+}(\Gamma)$. Then set $f_{\alpha}=f_{i j}$,

$$
Y(\Gamma)\left(f_{1}, \ldots, f_{n}\right):=\left(\bigotimes_{\alpha \in L^{+}(\Gamma)} f_{\alpha}\right) .
$$

We extend this definition by linearity if $f_{i} \in \mathrm{CH}^{n_{i}-1}(A, A)$ and $n_{i}=\left|\angle^{+}\left(c_{i}\right)\right|$; otherwise, if the condition is not met, we set $Y(\Gamma)\left(f_{1}, \ldots, f_{n}\right)=0$. 


\section{Extension to the boundary and dg properties}

Before we start the discussion of the dg-properties, we wish to point out the following. Let $A$ be a Frobenius algebra and let $\phi: A \rightarrow \check{A}$ be the isomorphism defined by the metric. If $\mu^{\dagger}$ is the adjoint of $\mu$ then $\phi \mu^{\dagger} \phi^{-1}=\check{\mu}:=\Delta$, i.e., the natural coproduct on $\breve{A}$. Moreover $\phi$ induces an isomorphism $\psi: T A \rightarrow T \check{A}$. Notice however, this is not an isomorphism of dg algebras, since $\phi \mu \phi^{-1}$ is the induced multiplication on $\check{A}$ whereas the natural differential comes from its co-simplicial structure given by $\Delta$. We will elaborate on this a little.

As we have discussed (see Lemma 3.5) for a Frobenius algebra there are canonical isomorphism $T A \simeq T \check{A} \simeq \mathrm{CH}^{*}(A, \check{A}) \simeq \mathrm{CH}^{*}(A, A) \simeq H_{\text {cyc-bar }}^{*}(A)$. Furthermore the dg structures of the middle two are compatible if $A$ is a Frobenius algebra yielding isomorphisms: $\operatorname{HH}^{*}(A, A) \simeq \mathrm{HH}^{*}(A, \check{A})$. So we can work with $T \check{A}$ or $T A$ to define the correlators. In the same vein also the spaces $\operatorname{Hom}\left(T A^{\otimes n}, T A^{\otimes m}\right)$ and $\operatorname{Hom}\left(T A^{\otimes n} \otimes T A^{\otimes m}, k\right)$ are isomorphic. Thus we can also work in the cyclic setting for defining the correlators.

A slight complication arises when we would like to check the dg-properties of the operadic of PROPic actions defined by dualizing say $m$ factors of $T A$ as above. The complication is that although the spaces $\operatorname{Hom}\left(T A^{\otimes n}, T A^{\otimes m}\right)$ and $\operatorname{Hom}\left(T A^{\otimes n} \otimes T A^{\otimes m}, k\right)$ are isomorphic if $A$ is Frobenius, they have different dg structures when they are endowed with the natural Hom differentials. In the first case $\partial_{\text {Hom }}(f)=f \circ \partial_{T A^{n}} \mp \partial_{T \check{A}^{m}} \circ f$, while in the second case we obtain that $\partial_{\text {Hom }}(\tilde{f})=f \circ \partial_{T A^{n+m}}$ where $\tilde{f}$ the image of $f$ under the isomorphism induced by $\phi$. As discussed above, these differentials are different. So to get a structure of a dg algebra over a dg-PROP, we have to additionally a priori declare some boundary inputs and other outputs. Now on the other hand, in the geometric models we are considering, say $\mathcal{A} r c^{i / o}$, the differential is a topological differential, which is independent of the discrete structure labelling the boundary. This independence of the boundary being labelled "in" or "out" will be the case for all topological models of surfaces, since these structures are naturally cyclic and the same type of argument applies. So we will have to be careful about the type of statements we can make. We can only expect a compatibility of the dg structure of the topological chains with the algebraic model if the discrete data of In and Out is canonical. When such a canonical operadically closed choice of In and Out is present, we indeed find the compatibility of the dg structures.

There is yet another caveat, though. An algebraic complex like the Hochschild complex does not "see" the moduli space structure in the sense that it does not naturally distinguish any differentials (say in the co-simplicial setup). In the moduli space case or the case of an open subset $\mathcal{A}$ of $\mathcal{A}^{L}$, however, certain differentials are set zero, since we are dealing with relative chains. In these situations, the Hochschild differential will force us to go to the boundary. This type of problem is present for higher genus 
and for several "ins" and "outs". In genus zero with only one output it does not appear, which explains the naturality of the operations of [K4], [K3], that is, in the case of Deligne's conjecture and the cyclic Deligne conjecture. In the general case, we have to grade the subspaces of $\mathscr{H o m}(T A)$ and pass to the associated graded to obtain the desired operations.

\section{1. $D g$ properties of the PROP action}

4.1.1. The tree level operads. If we fix one output boundary and chose the natural embedding of $\mathcal{T r e e}_{c p}$ into $\overline{\mathcal{A r c}}^{i \leftrightarrow o}$, then we are in the case of [K4], [K2] and the action is indeed the dg action discussed for $\mathscr{L} \mathcal{T r e e}_{c p}$ and $\mathcal{T}_{r e e_{c p}}$, respectively. ${ }^{5}$

4.1.2. The Sullivan chord diagram case. In the case of $\operatorname{Gr} \bigcup_{o}^{*}\left(\mathcal{A} r c^{i \leftrightarrow o}\right)$ with the conventions of [K5] reviewed in the Introduction, we can again put ourselves into the setting of a dg action of a dg-PROP on a dg algebra, but we need to extend the action to the boundary. Generalizing the arguments of [K4], [K2] we will show that the differential on the Hochschild side corresponds to the differential of $\mathcal{A}$ restricted to $\overline{\mathcal{A} r c}^{i \leftrightarrow o}$. If one removes an arc from a graph indexing a cell in $\mathrm{Gr} \mathcal{C}_{o}^{*}\left(\mathcal{A} r c^{i \leftrightarrow o}\right)$, it is not true in general that we still obtain a graph indexing a cell of $\mathrm{Gr}_{0}^{*}\left(\mathcal{A} r c^{i \leftrightarrow o}\right)$. This does not hold even if the genus is zero, so we will have to extend the action to

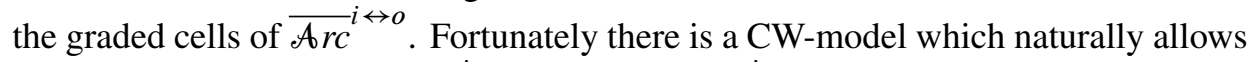
us to do this, that is, $C C_{*}\left(\overline{\mathcal{A r C}}_{1}^{i \leftrightarrow o}\right) \cong \mathrm{Gr} \mathcal{C}_{o}^{*}\left(\overline{\mathcal{A} r C}^{i \leftrightarrow o}\right)$.

We will see that in order to achieve a well-defined action, we will need some additional assumptions. These are satisfied if we restrict our attention to a commutative Frobenius algebra $A$.

Assumption: For the rest of the discussion of this subsection let $A$ be a commutative Frobenius algebra.

4.1.3. Extending to the boundary. The definition of this extension is dictated by the dg condition. This means that we will have to consider elements in A $r c$ which are in the limit of elements of $\mathcal{A} r c_{\#}^{0}$. Removing an arc corresponds to gluing together two polygons, and so we have to deal with not only polygons but also with cylinders and so forth. For a cylinder $C(n, m)$ with two boundaries given by polygons $p_{1} \in \operatorname{Poly}(n)$ and $p_{2} \in \operatorname{Poly}(m)$, and a choice of cut indices $(i, j)$, see Figure 6 for an example, we define

$$
\begin{aligned}
& Y(C(n, m),(i, j))\left(a_{1}, \ldots, a_{n}, b_{1}, \ldots, b_{m}\right) \\
& \quad:=\int \sum a_{1} \ldots a_{i}^{(1)} b_{j} \ldots b_{m} b_{1} \ldots b_{j-1} a_{i}^{(2)} \ldots a_{n},
\end{aligned}
$$

\footnotetext{
${ }^{5}$ See [K5] for the definitions of these subsets.
} 

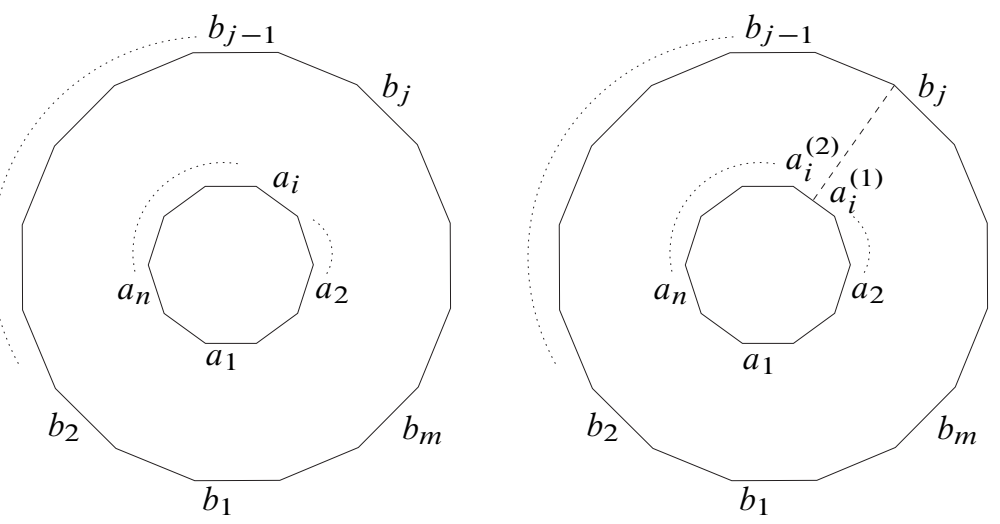

Figure 6. A cylinder with boundary components labelled by elements of $A$ and an indicated cut.

where $\Delta\left(a_{i}\right)=\sum a^{(1)} \otimes a^{(2)}$ is the co-product of $a_{i}$ using Sweedler's notation.

This definition is forced on us if we wish to ensure compatibility with the Hochschild differentials.

Remark 4.1. In order to ensure that equation (4.1) is independent of the choice of cutting edge, that is, independent of the indices $i$ and $j$ in equation (4.1), we assumed commutativity.

Lemma 4.2. If $A$ is a commutative Frobenius algebra then the right-hand side of equation (4.1) is independent of the choice of $i$ and $j$ and coincides with

$$
Y(n, m)=\int a_{1} \ldots a_{n} b_{1} \ldots b_{m} e,
$$

where $e=\mu \circ \Delta(1)$ is the Euler element.

Proof. If $A$ is commutative, then we have $Y(C(n, m))\left(a_{1}, \ldots, a_{n}, b_{1}, \ldots, b_{n}\right):=\int a_{1} \ldots a_{i-1}(\mu \circ \Delta)\left(a_{i}\right) a_{i+1} \ldots a_{n} b_{1} \ldots b_{m}$, but in any commutative Frobenius algebra one has

$$
\begin{aligned}
\int(\mu \circ \Delta)(a) b c & =\langle(\mu \circ \Delta)(a), b c\rangle \\
& =\langle a,(\mu \circ \Delta)(b c)\rangle=\langle a, b(\mu \circ \Delta)(c)\rangle=\int a b(\mu \circ \Delta)(c) .
\end{aligned}
$$

Fixing $a=a_{i}, b=\prod_{k \neq i} a_{i} \prod b_{j}$ and $c=1$, the claim follows. 
4.2. Correlators for $\mathcal{A}^{L}$. In general we extend the action as follows. Notice that given an arc graph $\alpha$ each complementary region $S \in \operatorname{Comp}(G)$ has the following structure: it is a surface of some genus $g$ with $r \geq 1$ boundary components whose boundaries are identified with a $2 k$-gons. Alternating sides belong to arcs and boundaries as above, and the sides come marked with 1 or 0 by identifying them with the angles of the underling arc graph. Now let $\operatorname{Sides}^{\prime}(S)$ be the sides which have an angle marking by 1 and let $\chi$ be the Euler characteristic of $S$. We set

$$
Y(S)\left(\bigotimes_{s \in \operatorname{Sides}^{\prime}(S)} a\right):=\int\left(\prod_{s \in \operatorname{Sides}^{\prime}(S)} a_{s}\right) e^{-\chi+1},
$$

where $e:=\mu(\Delta(1))$ is the Euler element. For an angle marked partitioned arc graph $\alpha^{p}$ we set

$$
Y\left(\alpha^{p}\right)\left(\bigotimes_{S \in \operatorname{Comp}\left(\alpha_{i}\right)}\left(\bigotimes_{s \in \operatorname{Sides}^{\prime}(S)} a_{s}\right)\right)=\bigotimes_{S \in \operatorname{Comp}\left(\alpha_{i}\right)} Y(S)\left(\bigotimes_{s \in \operatorname{Sides}^{\prime}(S)} a_{s}\right) .
$$

For $\alpha \in C C_{*}\left(\mathcal{A}^{\llcorner}\right)$we simply set

$$
Y(\alpha)=Y(\mathcal{P}(\alpha))
$$

4.2.1. The Hochschild differential. Now consider the operation $Y\left(\alpha^{p}\right)$ for $\alpha \in \operatorname{Gr} \ell_{o}^{*}\left(\mathcal{A} r c_{\#}^{i \leftrightarrow o}\right)$. The Hom differential on this viewed as an element in $\operatorname{Hom}\left(\mathrm{CH}^{\mathrm{In}}(A, \check{A}), \mathrm{CH}^{\text {Out }}(A, \check{A})\right.$ is given by $\partial_{\mathrm{Hom}}(Y)\left(f_{i}\right)=Y\left(\partial_{C y c}\left(f_{i}\right)\right) \mp \partial_{C y c} Y\left(f_{i}\right)$. Here we index the tensor products by the sets In and Out and denote the differential of the cyclic bar complex by $\partial_{C y c}$.

We can consider $Y \in \operatorname{Hom}\left(\mathrm{CH}^{\mathrm{In}}(A, \check{A}) \otimes\left(\mathrm{CH}^{\text {Out }}(A, \check{A})^{\vee}, k\right)\right.$ by decorating the In boundaries with the elements $f_{i}$ and the Out boundaries by elements $a_{i} \in A$. That is, $Y\left(f_{0}, \ldots, f_{n}\right)\left(\otimes a_{i}\right)$. Then the first term in the differential is given by applying $\Delta=\check{\mu}$ cyclically to each element $f_{i}$ on the left-hand side, viewed as an element in $T \check{A}$ decorating the In boundaries. The second term in the differential is given by the sum obtained by decorating exactly one of the angles of the Out boundaries with the product of two variables $a_{i} a_{i+1}$. These summands will cancel with summands from the first term essentially due to the Frobenius condition

$$
\left\langle f_{i j}, a_{k} a_{k+1}\right\rangle=\left\langle\Delta\left(f_{i}^{j}\right), a_{k} \otimes a_{k+1}\right\rangle,
$$

where we write $f_{i j}$ using the notation of 3.2.2. Hence, we are left with the summands of the first term that are not cancelled. These correspond to angles on the Out boundaries marked by 0 .

More precisely, consider decorating two neighboring angles at an In boundary by say $\Delta\left(f_{i j}\right)$, where the common edge $e$ belongs to an angle marked by 0 on the Out boundary. There are two cases. Either the edge is separating, that is, it separates two 
different complementary regions, or it is non-separating, that is, the same region lies on both sides of the edge; see also Figure 7.

First let us consider the angle markings all given by 1 . Let $P_{1}$ and $P_{2}$ be the two complementary regions on the two sides of the edge $e$. Fix the notation $a_{1}, \ldots, a_{n}$ for the elements decorating the sides of the polygon $P_{1}$, and $b_{1}, \ldots, b_{m}$ for the elements decorating the sides of $P_{2}$, where in both cases the enumeration is compatible with the cyclic order. Also set $\Delta\left(f_{i}^{j}\right)=\sum a^{(1)} \otimes b^{(2)}$ in Sweedler's notation. Say that $a^{(1)}=a_{1}$ and $b_{1}=b^{(2)}$; see Figure 7 .

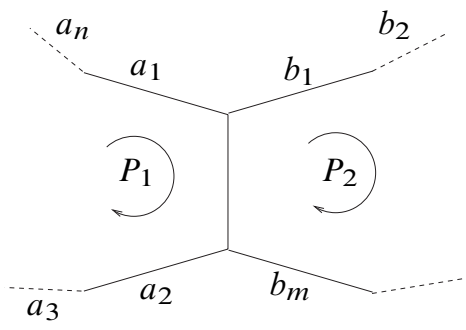

I

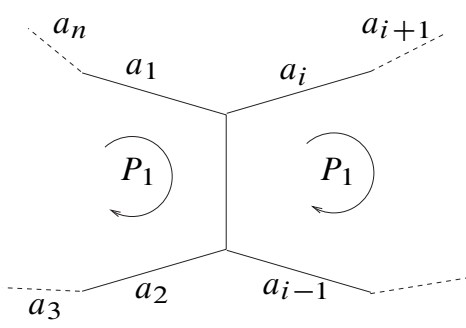

II

Figure 7. I. The separating case. II. The non-separating case.

In the separating case applying the Hochschild differential before applying $Y$, we obtain a contribution of the type

$$
\sum \int a^{(1)} a_{2} \ldots a_{n} \cdot \int b^{(2)} b_{2} \ldots b_{m}=\int f_{i}^{j} b_{2} \ldots b_{m-1}\left(b_{m} a_{2}\right) a_{3} \ldots a_{n},
$$

which is the contribution obtained by gluing the two polygons along the common edge and decorating one of the two joined sides by $f_{i}^{j}$ and the other by $b_{m} a_{2}$.

In the non-separating case, we only have one complementary region $P_{1}$; let $a_{1}, \ldots, a_{m}$ be the elements decorating the sides. Also again use $\Delta\left(f_{i}^{j}\right)=$ $\sum a^{(1)} \otimes a^{(2)}$, let $a_{1}=a^{(1)}$, and put $a_{i}=a^{(2)}$. The contribution reads

$$
\begin{aligned}
\pm \int a^{(1)} a_{2} \ldots a_{i-1} a_{i}^{(2)} a_{i+1} \ldots a_{n} & =\int(\mu \circ \Delta)\left(f_{i}^{j}\right) a_{2} \ldots a_{i-1} a_{i+1} \ldots a_{n} \\
& =\int f_{i}^{j} a_{3} \ldots a_{i-1} a_{i+1} \ldots\left(a_{n} a_{2}\right) e,
\end{aligned}
$$

where $e=\mu \circ \Delta(1)$.

First if both polygons are quadrangles with all markings 1 , then the term in equation (4.7) cancels with the Hochschild differential on $Y$ as discussed above. In all other cases, if $\alpha$ is an quasi-filling element of $\overline{A A r c}^{i \leftrightarrow o}$ endowed with the standard angle markings, then one of the elements $b_{m}, a_{2}$ is one in equation (4.7), so that we indeed 
obtain the contribution to the correlation function associated to the surface in which the arc has been removed. In the same situation but with a separating arc in equation (4.8) either $a_{2}$ or $a_{m}$ is equal to 1 , and we again obtain the contribution to the correlation function associated to the surface in which the arc has been removed - now basically by definition.

This calculation generalizes to arbitrary $Y\left(\alpha^{p}\right)$ for $\alpha \in \operatorname{Gr} \mathcal{C}_{o}^{*}\left(\overline{\mathcal{A} r c}^{i \leftrightarrow o}\right)$.

Proposition 4.3. The dg structures of $C C_{*}\left(\overline{\mathcal{A r c}}_{1}^{i \leftrightarrow o}\right)$ and $\operatorname{Hom}(\mathrm{CH}(A, A)$ are respected by $Y$. Or in other words: The equation (4.3) and hence the equation (4.4) are the operations corresponding to the summands of the Hom differential of $Y\left(\alpha^{p}\right)$, and furthermore these summands correspond to the respective boundary components of $\alpha$.

Proof. In the case of splitting angles, we see by the considerations above that there are two terms in the Hom differential which cancel. In fact all of the terms $\partial \circ Y$ cancel in this way. On the other hand generalizing the formalism explained above, we see that when two neighboring angles which are not parallel are assigned the coproduct of an element $a_{i}$, the resulting operation is the operation associated to the arc graph in which the arc corresponding to the common edge is removed. This either causes two bordering complementary regions to be joined or self-glues a complementary region to itself. In both cases the resulting function is the product of integrals over all the boundaries of the joined surface. In the case of self-gluing this yields a term $\mu\left(\Delta\left(a_{i}\right)\right)$. As in Lemma 4.2, we can "transfer" the $\mu \circ \Delta$ to an inserted unit. Now iterating this process, we have to remove $-\chi+1$ edges to obtain a complementary region $S$ with genus $g$ and $r$ boundary components. This accounts for the tensor power of $e$. Also iterating the argument of the "transferring" the $\mu \circ \Delta$ from the elements $a_{i}$ to 1 one obtains the independence of the exact incidences of the removed edges. That is, if the surface $S$ can obtained by removing other edges, the resulting operation will be the same. This means that such an expression in the differential is well defined.

Collecting the results, we find:

Theorem 4.4. The $Y(\alpha)$ defined in equation (4.5) give operadic correlation functions for $C C_{*}\left(\overline{A A r c}_{1}^{i \leftrightarrow o}\right)$ and induce a dg action of the dg-PROP $C C_{*}\left(\overline{\mathcal{A r r c}}_{1}^{i \leftrightarrow o}\right)$ on the dg algebra $\overline{\mathrm{CH}}^{*}(A, A)$ of reduced Hochschild co-chains for a commutative Frobenius algebra $A$.

The $Y(\alpha)$ also yield correlation functions on the tensor algebra of the co-cycles of a differential algebra $(A, d)$ over $k$ with a cyclically invariant trace $\int: A \rightarrow k$ that satisfies $\int d a=0$ and whose induced pairing on $H=H(A, d)$ turns $H$ into a Frobenius algebra. These correlations functions are operadic chain level correlation functions. 
Proof. We have shown in [K5] that the $C C_{*}\left(\overline{A A r c}_{1}^{i \leftrightarrow o}\right)$ form a dg-PROP that is isomorphic to the dg-PROP $\operatorname{Gr}^{*} \mathcal{C}_{o}^{*}\left(\overline{\mathcal{A r c}}^{i \leftrightarrow o}\right)$, and we have that the map $\mathcal{P}^{<}$is operadic/PROPic. Now the gluing for the correlation functions coincides with the algebraic one used to define the PROP structure of Proposition 1.20. On the Hochschild side, we simply plug in elements. On the graph side, we correspondingly plug in angles marked with 1 to angles marked with 1 . The first thing we have to make sure is that the steps (1) and (2) in Definition 1.18 are respected on the Hochschild side. By [K3] the double twisted case of step (1) corresponds to applying the Connes operator $B$ to both sides, that is, applying $B^{2}$, and hence yields zero. The case of (2) cannot occur since the arcs are only running from In to Out. Therefore the gluing actually corresponds to the "twisted" gluing. Lastly, in the gluing for $\mathcal{P} \angle \overline{\mathcal{A}} \boldsymbol{~} r \mathrm{i}^{i \leftrightarrow o}$ there are no terms of lower degree because one never glues separating to separating angles, since these are labelled by 0 on the Out boundaries and by 1 on the In boundaries. So the gluing on the Hochschild side corresponds to the gluing in the associated graded for the non-partitioned graphs before applying $\mathcal{P}^{\angle}$.

The last statement follows from Proposition 2.6

Remark 4.5. Seemingly related results have been obtained by [TZ] in a different setting. Their definition of Sullivan chord diagrams is, however, different from ours and, as far as we can see, also the definition of the action differs. It is therefore not possible to relate their calculations to the present ones or those of [K2], [K4] directly. It would be interesting to know how if it is possible to compare the two actions despite their different settings.

Corollary 4.6. The operadic correlation functions descend to give a PROP action of $H_{*}\left(\overline{A A r c}_{1}^{i \leftrightarrow o}\right)$ on $\mathrm{HH}^{*}(A, A)$ for a commutative Frobenius algebra $A$.

\subsection{Co-simplicial properties of the action of moduli space}

4.3.1. The operation of $\overline{\mathscr{L A A r}}^{i} \leftrightarrow$ o. We recall from [K5] that $\overline{\mathscr{L} A r c}^{i \leftrightarrow o}$ is the subspace of $\overline{\mathcal{A} r c} i \leftrightarrow o$ whose underlying arc graphs are not twisted at the In boundaries. Using the constant marking $m k^{L} \equiv 1$ this space is a subspace of $\mathcal{A}^{L}$.

Proposition 4.7. The correlation functions (4.5) are operadic correlation functions for the PROP $\mathcal{C}_{o}^{*}\left(\overline{\mathscr{L} A r c}^{i \leftrightarrow o}\right)$, the tensor algebra on the co-cycles $Z(A)$ of a quasiFrobenius algebra. That is, they give chain level correlators.

Proof. The algebraic operadic composition on the level of partitioned angle marked arc graphs corresponds to the insertion on the $\mathscr{H o m}(T Z(A))$ side. The conditions for the twisted gluing needed to make $\mathcal{P}^{<}$operadic never occur. There are never any double twisting and also never any closed loops. 
4.3.2. The tree level: Tree. As we have previously discussed, one cannot expect that the cyclic operad will go over to the dg setting. We, however, have the following interesting observation.

Lemma 4.8. Using the isomorphism $\mathrm{CH}(A, A) \simeq \overline{T A}$ for a Frobenius algebra, the operations defined by the cells of $\mathcal{T}$ ree embedded into $A$ Ar $c_{\#}^{\swarrow}$ by the constant marking $m k^{<} \equiv 1$ yields the operations $\sqcup$ and $\square$ of [MS3]. In particular, the operation of $\overline{\mathscr{L} A r c}^{i \leftrightarrow o}$ induces the $\Xi_{2}$ operation of [MS3].

Proof. This is a straightforward verification. The relevant arc families are depicted in Figure 8.
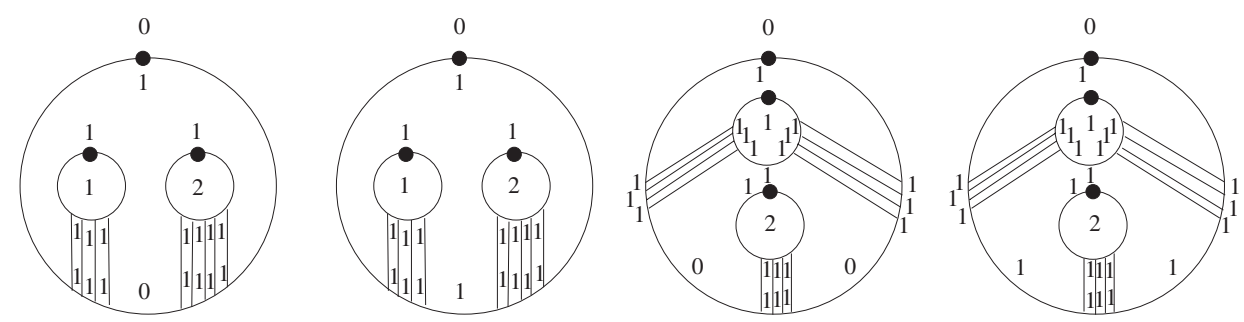

Figure 8. Examples of the angle marked partitioned families yielding $\cup, \sqcup,{ }_{i}$ and $\square_{i}$.

Remark 4.9. In other words using the language of [MS3], our operations allow us to recover the operation of the functor operad given by the Kan extension of the operad of sequences with differentials up to complexity 2 . This action corresponds to the genus-0-LL Tree operad embedded by the marking $m k^{L} \equiv 1$. In general, even going to the boundary as in the last paragraph, we cannot expect to get actions of the higher $\Xi_{n}$. This is commensurate with Deligne's conjecture. Since in the differential structure of the sequences of higher complexity one does not retain the topological information of the underlying surface when moving to the boundary, one would, in contrast to the last paragraph, have to identify the surfaces $S$ again with polygons, which is not true in $\mathcal{A} r c$, but can be done in $8 t$ A $r c$, the stabilization of the arc operad.

Two questions arise in this setting. What are the conditions to get the higher differentials? Is there a co-simplicial type of setup for $\mathcal{A} r c_{\#}^{0}$ or $\mathcal{A} r c_{\#}^{0, L}$ ? The first question will be addressed in [K6] where we will deal with the stabilization of the arc operad and its loop structure.

4.4. New monoidal structures on the Hochschild co-chains. In order to match the geometric grading by non-partitioning angles on the algebraic side, we will introduce a new monoidal structure of $A$-bi-modules, where $A$ is an associative ring. This 
structure will allow us to define a natural grading on a subcomplex of the Hochschild complex whose associated graded is the correct receptacle of our correlation functions.

Definition 4.10. Let $A$ be an associative ring and let $M$ and $N$ be $A$-bi-modules. We define $M \otimes N$ to be $M \otimes A \otimes N$ with the obvious structure of $A$-bi-module.

4.4.1. A new co-product. If we study the basic operations on the Hochschild cochains, which are a generalization of the operations of $\mathcal{S}$ to the brace suboperad Brace, we see that they naturally correspond to slightly different operations induced by the new monoidal structure.

We will first treat the operad corresponding to $\mathcal{S}$. For this we notice that with the monoidal structure $\otimes$ on $T A$ the product is a map $\otimes: T A \otimes T A \rightarrow T A$ and dually the co-product is a map $\diamond: T A \mapsto T A \otimes T A$. Here the first map is given by $\left(a_{1} \otimes \cdots \otimes a_{n}\right) \otimes b \otimes\left(c_{1} \otimes \cdots \otimes c_{m}\right) \mapsto a_{1} \otimes \cdots \otimes a_{n} \otimes b \otimes c_{1} \otimes \cdots \otimes c_{m}$, that is, it "raises degree by one"; and the second map is given by $a_{1} \otimes \cdots \otimes a_{n} \mapsto$ $\sum_{i} \pm\left(a_{1} \otimes a_{i-1}\right) \otimes a_{i} \otimes\left(a_{i+1} \otimes \cdots \otimes a_{n}\right)$.

Now the multiplication $\cup$ in $\mathrm{CH}^{*}(A, A)$ is given by the multiplication $\otimes$ in the tensor algebra $T \check{A}$, and the multiplication $\mu$ of the algebra $A$ :

$$
\cup: A \otimes \check{A}^{\otimes n} \otimes A \otimes \check{A}^{\otimes m} \stackrel{\otimes \circ \sigma}{\longrightarrow} A \otimes A \otimes \check{A}^{\otimes n+m} \stackrel{\mu}{\longrightarrow} A \otimes \check{A}^{\otimes n+m} .
$$

Here $\sigma$ is just the permutation of the tensor factors and $f \cup g\left(a_{1}, \ldots a_{n}, b_{1}, \ldots b_{m}\right)=$ $f\left(a_{1}, \ldots, a_{n}\right) g\left(b_{1}, \ldots, b_{m}\right)$.

Using the new monoidal structure for the same canonical maps we obtain a new multiplication

$$
\sqcup:\left(A \otimes \check{A}^{\otimes n}\right) \otimes\left(A \otimes \check{A}^{\otimes m}\right) \stackrel{\bigotimes \circ \sigma}{\longrightarrow} A \otimes A \otimes\left(\check{A}^{\otimes n} \otimes \check{A}^{\otimes m}\right) \stackrel{\mu}{\longrightarrow} A \otimes \check{A}^{\otimes n+1+m} .
$$

Here $\sigma$ is again just the permutation of the tensor factors and

$$
f \sqcup g\left(a_{1}, \ldots a_{n}, b, c_{1}, \ldots c_{m}\right)=f\left(a_{1}, \ldots, a_{n}\right) b g\left(c_{1}, \ldots, c_{m}\right) .
$$

This is exactly the operation induced by the co-simplicial structure used in [MS3], which we recover using the embedding $m k^{L} \equiv 1$.

In the setting of operadic correlation functions, we are using the coproduct to separate the different tensor factors of $\breve{A}$ at the different boundaries before integrating over them. This means that in the current setting, we should again use the co-product $\diamond$. Each time we use the new co-product $\diamond$ this has the effect of inserting a tensor factor of $A$, so that, for instance, the usual $\circ_{i}$ operations of $\mathrm{CH}^{*}(A, A)$ which use two co-products become operations $\square_{i}$ where

$$
\begin{aligned}
& \square_{i}(f, g)\left(a_{1}, \ldots, a_{n+m+2}\right) \\
& \quad=f\left(a_{1}, \ldots, a_{i-1}, a_{i} g\left(a_{i+1}, \ldots a_{i m}\right) a_{i+m+1}, a_{i+m+2}, \ldots, a_{n+m+2}\right) .
\end{aligned}
$$

This is again the operation obtained by [MS3]. 
4.5. Graded correlators. In general from the correlators of $\alpha^{p}$ whose underlying $\operatorname{arc}$ graph $\alpha$ is in $\bigcup_{o}^{*}\left(\mathcal{A} r c_{\#}(F)\right)$ we obtain maps

$$
Y\left(\alpha^{p}\right): T A^{\otimes n} \rightarrow \bigotimes_{i=1}^{n} A^{\otimes n_{i}+1} \rightarrow k .
$$

Notation 4.11. If $k=|E(\alpha)|$, and $p \in P(n, k)$, we will use the following notation: $\diamond^{l}: T A \rightarrow T A^{\otimes l+1}$ is the iteration of $\diamond$ given by $\left(\diamond \otimes\left(\mathrm{id}_{A} \otimes i d_{T A}\right)^{\otimes l}\right) \circ \diamond \otimes$ $\left.\left(\mathrm{id}_{A} \otimes i d_{T A}\right)^{\otimes l-1}\right) \circ \ldots \circ\left(\diamond \otimes \mathrm{id}_{A} \otimes \mathrm{id}_{T A}\right) \circ \diamond$.

We have

$$
Y\left(a^{p}\right)=\left(\bigotimes_{\pi \in \operatorname{Comp}\left(\alpha^{p}\right)} Y_{\mathrm{Poly}_{2}}(\pi) \otimes \bigotimes_{i=1}^{k} \eta^{n_{i}-1}\right) \circ \sigma \circ \bigotimes_{i=0}^{n} \diamond^{n_{i}},
$$

where we think of the complementary regions $\operatorname{Comp}(\alpha)$ as a subset of the complementary regions of $\alpha^{p}$, and $Y_{\mathrm{Poly}_{2}}$ are the polygon correlation functions defined in equation 2.11. Here $\sigma$ permutes the factors of $T A$ and the factors of $A$ corresponding to $\bigotimes_{i=1}^{n} T A^{\bigotimes n_{i}+1}$, and we use Notations 2.10 and 4.11.

Definition 4.12. We let $\mathcal{M} \mathcal{S}(n) \subset \operatorname{Hom}(T A)(n)$ be the image of all the operations of $\mathcal{P} \angle \mathcal{A} r c_{\#}(n)$ by considering 0 as "out".

We get an analogous statement to the Proposition 2.11.

Proposition 4.13. After dualizing to obtain elements in $\operatorname{Hom}\left(T A^{\otimes n+1}, k\right)$ any element in $\mathcal{M}$ C $S(n)$ can be written uniquely as in equation (4.14). Set $l=\frac{1}{2} \sum_{i}\left(n_{i}+1\right)-1$. Then $\mathcal{M C} S$ is graded by $l$. Moreover, the composition in $\mathcal{M} C S$ respects the induced filtration of elements of degree $\leq l$. Finally, the decomposition identifies $\mathcal{M} \mathcal{S}$ with the subspace of $\operatorname{Hom}(T A)$ obtained by dualization for the subspace generated by the coproduct $\diamond$, permutations of the factors $A$ and $T A$, and $\eta$ in $\bigoplus_{n} \operatorname{Hom}\left(T A^{\otimes n+1}, k\right)$.

Proof. The proof is analogous to the proof of Proposition 2.11. The first statement is again clear by the definition of $\mathcal{M} \mathcal{S}$ as the image. Likewise, the last statement is again straightforward by arranging the operation in the specified order. On the other hand it is easy to give the arc graph in $\mathcal{P} \mathcal{A} r c_{\#}^{0, L}(n)$ by drawing one arc for each factor of $\eta$ with the incidence relations given by $\sigma$. One quick way is to dually draw the partitioned ribbon graphs with one vertex per complementary region. This identifies the two subspaces. In this identification there is one factor of $\diamond$ for each inner angle which is not partitioning. The last claim that the operations respect the filtrations is clear after identifying $k$ with the dimension of the cell, that is, the number of edges minus one, of the underlying graph for the operation. The mentioned equality follows 
from the combinatorial identity $\left|L_{\text {inner }}\right|+\mid \angle$ outer $|=|$ Flags $|=2|$ edges $\mid$, which still holds true.

Proposition 4.14. For any Frobenius algebra $A$ the correlations functions of equation (4.14) define operadic correlation functions for $\mathrm{Gr} \mathcal{P} \angle \mathcal{A} r c_{\#}$ with values in the associated graded $\mathrm{Gr} \mathcal{M} \mathcal{S}$ of $\mathcal{M} \mathcal{C} \subset \subset \operatorname{Hom}(T A)$. By regarding $\alpha \rightarrow Y(\mathcal{P}<\alpha)$ the same statement hold also for $\mathrm{Gr}_{o}^{*}\left(\mathcal{A} r \mathrm{C}^{\llcorner}\right)$.

Proof. Using the Casimir element to dualize, we only have to show that the resulting structure is that of an algebra over a cyclic operad. Again the $\mathbb{S}_{n+1}$ equivariance is manifest. After dualizing the gluing on the flags in the operadic composition turns into the identity map id: $A \rightarrow A$, so that indeed the gluing ${ }_{i}$ on $\mathcal{P}^{<} \mathcal{A} r c_{\#}$ maps to insertion at the $i$-th place in $\operatorname{Hom}(T A)$. Dealing with the extra steps (1) and (2) in the definition of the gluing in $\mathcal{P}^{\angle} \mathcal{A} r C_{\#}$, we see that on the side of $\operatorname{Hom}(T A)$ they would not yield zero. However in Gr $\mathcal{M} \mathcal{S}$ both these cases are projected out, since they correspond to operations of lower degree. The same is true for both $\mathrm{Gr} \mathcal{P} \angle \mathcal{A} r c_{\#}^{0, \angle}$ and $\mathrm{Gr} \mathcal{C}_{o}^{*} A \mathcal{A} r c_{\#}^{0, \angle}$ by definition.

Defining the action on the Hochschild complex through the tensor algebra as in \$3.2.2, we obtain:

Theorem 4.15. Let $A$ be a Frobenius algebra and let $\mathrm{CH}^{*}(A, A)$ be the Hochschild complex of the Frobenius algebra. Then the cyclic chain operad of the open cells of A $r^{\perp}$ act on $\mathrm{CH}^{*}(A, A)$ via correlation functions. Hence so do all the suboperads, subdioperads and PROPs of [K5] mentioned in the introduction. In particular the graph complex of $M_{g, n+1}^{1^{n+1}}$, the Moduli space of pointed curves with fixed tangent vectors at each point, act on $\mathrm{CH}^{*}(A, A)$ by its two embeddings into $A$ orc $c_{\#}^{0, L}$. Furthermore, on $\mathcal{P}^{<} \mathcal{A} r c_{\#}^{0}$ the correlation functions are operadic correlation functions with values in $\mathrm{Gr} \mathcal{M}$ CS. Moreover, the operations of the suboperad $\mathcal{T}_{\text {ree }} \mathrm{e}_{\mathrm{p}}$ correspond to the operations $\sqcup$ and $\square_{i}$ induced by $\Xi_{2}$ as defined in [MS3].

The same formula, equation (4.14), also yields operadic correlation functions for the tensor algebra of the co-cycles of a differential algebra $(A, d)$ over $k$ with a cyclically invariant trace $\int: A \rightarrow k$ which satisfies $\int d a=0$ and whose induced pairing on $H=H(A, d)$ turns $H$ into a Frobenius algebra, i.e., they are chain level operadic correlation functions with values in $\mathrm{Gr} \mathcal{M}$ C 8 .

Proof. We use operadic correlation function $Y$ above to give maps $\mathrm{CH}^{p_{1}} \otimes \cdots \otimes$ $\mathrm{CH}^{p_{n+1}} \simeq \check{A}^{\otimes p_{1}+1} \otimes \cdots \otimes \check{A}^{p_{n}+1} \rightarrow k$. All the necessary properties follow from Propositions 4.8 and 4.14. The last statement again follows from Proposition 2.6. 
4.6. Application to string topology. Let $M$ be a simply connected compact manifold $M$, denote the free loop space by $\mathscr{L} M$, and let $C_{*}(M)$ and $C^{*}(M)$ be the singular chains and (co)-chains of $M$. We know from [J], [CJ] that $C_{*}(\mathscr{L} M)=$ $\mathrm{CH}^{*}\left(C^{*}\left(M, C_{*}(M)\right)\right.$ and $H_{*}(\mathscr{L} M) \simeq \mathrm{HH}^{*}\left(C^{*}(M), C_{*}(M)\right)$. Moreover $C^{*}(M)$ is an associative dg algebra with unit, differential $d$ and an integral ( $M$ was taken to be a compact manifold) $\int: C^{*}(M) \rightarrow k$ such that $\int d \omega=0$. By using the spectral sequence and taking field coefficients we obtain operadic correlation functions $Y$ for Tree on $E^{1}=\mathrm{CH}^{*}(H, H)$, which converges to $\mathrm{HH}^{*}\left(C^{*}(M)\right)$ and which induces an operadic action on the level of (co)-homology. Except for the last remark, this was established in [K2].

Theorem 4.16. When taking field coefficients, the above action gives a dg action of a dg-PROP of Sullivan chord diagrams on the $E^{1}$-term of a spectral sequence converging to $H_{*}(L M)$, that is, the homology of the loop space a simply connected compact manifold, and hence induces operations on this loop space.

Proof. Recall from [CJ] that the isomorphism $C_{*}(\mathscr{L} M)=\mathrm{CH}^{*}\left(C^{*}\left(M, C_{*}(M)\right)\right.$ comes from dualizing the isomorphism $C_{*}(\mathscr{L} M)=\mathrm{CH}_{*}\left(C^{*}(M)\right)[\mathrm{J}]$. Calculating the latter with the usual bi-complex [L], we see that the $E^{1}$-term is given by $\mathrm{CH}_{*}\left(H^{*}(M)\right)$, and dualizing the corresponding $E^{1}$ spectral sequence we obtain that $\mathrm{CH}^{*}\left(H^{*}(M), H_{*}(M)\right)$, so we get an operation of the $E^{1}$ level. Since the operation of $\mathcal{T}$ ree is $\mathrm{dg}$, it is compatible with the $E^{1}$ differential and hence gives an action on the convergent spectral sequence computing $H_{*}(\mathscr{L} M)$, and so on its abutment.

\section{Concluding remarks}

In this paper and its first part [K5] we have systematically used the A $r c$ operad and its cousins to give operations on the Hochschild co-chains of a Frobenius algebra by extending and building on our results of [K4], [K2]. In particular, we have given correlation functions for $\mathcal{A} r c_{\#}$. In physics terms this could be expected by using the logic of $[\mathrm{KR}]$ as follows. If the closed string states are thought of as deformations of the open string states and the open string states are represented by a category of $D$-branes, then the closed strings should be elements of the Hochschild co-chains of the endomorphism algebra of this category. Now thinking on the worldsheet, we can insert closed string states. That is, for a world sheet we should get a correlator by inserting, say $n$ closed string states. This is what we have done if one simplifies to a space filling $D$-brane and twists to a TCFT.

For string topology, we have given operations using the spectral sequence. So the question remains whether we loose any information by passing to the associated graded. This is indeed an interesting question. It seems though that since all the operations of string topology preserve the grading and not just the filtrations, we have 
not lost any information. A question that one could ask is how different possible lifts from the associated graded to the filtered complex are related. It is conceivable that an interesting "up to homotopy" structure is lurking, which may possibly be related to Frobenius manifold structures found, e.g., in [Me1]. An interesting observation in this respect is that the operadic correlation functions allow one to lift to at least the co-cycle level in the tensor algebra setting. Perhaps this gives enough information to compare the two sets of operations. It seems that compared with $[\mathrm{CJ}]$ the operations should even be the same. Although a priori they might differ, the operation of [CJ] do not only respect the filtration, but they act with a definite bi-degree in the bi-grading and hence $a$ posteriori seem to have no lower order contributions in the filtration.

It seems that the combinatorial version of the moduli space of [P2], [KLP] is particularly suited for these applications. One amazing coincidence is that the Hochschild differential forces one to consider Penner's compactification. That is, it forces to move from $\mathcal{A} r c^{i \leftrightarrow o}$ to $\overline{\mathcal{A} r c}^{i \leftrightarrow o}$. Another interesting remark is that the grading by the number of arcs on the Hochschild side is reminiscent of the operation of open strings rather than closed strings in the framework set up in [KP]. Here we have the additional restriction that there is exactly one arc per window in the terminology of [KP], and the $D$-brane label corresponds to dualizing the respective element labelling the marked point to live in $A$. This observation could be a "shadow" of the open/closed duality.

The interplay between algebra and geometry is astonishing. The algebra side for instance demands the insertion of degeneracies in order to obtain the BV-operator $B$. This manifests itself in the restriction to the PROP $\mathcal{A} r c^{i \leftrightarrow o}$ and the preservation of the algebraic dg structure on the geometric side then forces one to move to the boundary, viz. $\overline{\mathcal{A} r c} i \leftrightarrow o$ or the Sullivan chord diagrams.

This leads us to an interesting aspect which we did not cover, namely the cosimplicial setup for the Arc complex. That is, reverse engineering the Arc-complex by starting with a cosimplicial model coming from partitioned arc graphs. For the subset of $\mathscr{L} \mathcal{T}$ ree this is essentially what has been done in [MS3]. Hence one could expect that the totalization of the arising complex operates on the totalization of the relevant Hochschild complex. If this is possible one would have the hope of "dg compatibility" after passing to the totalization. One of the difficulties, however, is that there is more than one "output", so that one cannot directly use a co-simplicial structure since this relies on the category of maps, viz. several inputs, but only one output. In order to accommodate this one either has to break the cyclic setting of the cyclic operad, or one has to construct a suitable category of sets with correspondences.

Of course a generalization to the $A_{\infty}$ case would be very useful. The tree level version is worked out in [KSch]. A further area which deserves study are the implications for the operations on the cyclic co-chains and the associated $S^{1}$ equivariant theories, e.g., in the spirit of [W]. In our context, we can also simply to restrict to Connes' cyclic complex.

Finally, we wish to emphasize that at several points we had to avoid closed loops. 
On the topological side this basically comes from the cell decomposition of moduli space, which does not have any graphs with closed loops. On the Hochschild side this was not as natural. We avoided the occurrence, by either restricting the type of graph or passing to the associated graded. In the setting of partially measured foliations however these closed leaves are very natural. So one cannot help but wonder whether there is yet another generalization of this whole story to foliations, as outlined in the Appendix of [KP].

\section{References}

[BF] C. Berger and B. Fresse, Une décomposition prismatique de l'opérade de BarrattEccles. C. R. Acad. Sci. Paris Sér. I Math. 335 (2002), 365-370. Zbl 1016.18005 MR 1931518

[CS] M. Chas and D. Sullivan, String topology. Ann. of Math., to appear; preprint 1999, arXiv:math.GT/9911159

[Ch] D. Chataur, A bordism approach to string topology. Internat. Math. Res. Notices 2005 (2005), 2829-2875. Zbl 1086.55004 MR 2180465

[C] R. L. Cohen, Multiplicative properties of Atiyah duality. Homology Homotopy Appl. 6 (2004), 269-281. Zbl 1072.55004 MR 2076004

[CG] R. L. Cohen and V. Godin, A polarized view of string topology. In Topology, geometry and quantum field theory (Oxford, 2002), London Math. Soc. Lecture Note Ser. 308, Cambridge University Press, Cambridge, 2004, 127-154. Zbl 1095.55006 MR 2079373

[CJ] R. L. Cohen and J. D. S. Jones, A homotopy theoretic realization of string topology. Math. Ann. 324 (2002), 773-798. Zbl 1025.55005 MR 1942249

[CV] J. Conant and K. Vogtmann, On a theorem of Kontsevich. Algebr. Geom. Topol. 3 (2003), 1167-1224. Zbl 1063.18007 MR 2026331

[Co] K. J. Costello, Topological conformal field theories and Calabi-Yau categories. Adv. Math. 210 (2007), 165-214 Zbl 05132553 MR 2298823; The Gromov-Witten potential associated to a TCFT, preprint 2005, arXiv:math.QA/0509264; A dual version of the ribbon graph decomposition of moduli space. Geom. Topol. 11 (2007), 1637-1652. Zbl 1131.32008 MR 2350462

[H] J. L. Harer, Stability of the homology of the mapping class groups of orientable surfaces. Ann. of Math. (2) 121 (1985), 215-249. Zbl 0579.57005 MR 786348

[HM] J. Hubbard and H. Masur, Quadratic differentials and foliations. Acta Math. 142 (1979), 221-274. Zbl 0415.30038 MR 523212

[J] J. D. S. Jones, Cyclic homology and equivariant homology. Invent. Math. 87 (1987), 403-423. Zbl 0644.55005 MR 870737

[KLi1] A. Kapustin and Y. Li, D-branes in Landau-Ginzburg models and algebraic geometry. J. High Energy Phys. 2003, no. 12, 005. MR 2041170 
[KLi2] A. Kapustin and Y. Li, Topological correlators in Landau-Ginzburg models with boundaries. Adv. Theor. Math. Phys. 7 (2003), 727-749. Zbl 1058.81061 MR 2039036

[KR] A. Kapustin and L. Rozansky, On the relation between open and closed topological strings. Comm. Math. Phys. 252 (2004), 393-414. Zbl 1102.81064 MR 2104884

[K1] R. M. Kaufmann, Operads, moduli of surfaces and quantum algebras. In Woods Hole mathematics. Ser. Knots Everything 34, World Scientific, Singapore 2004, 133-224. MR 2123369

[K2] R. M. Kaufmann, A proof of a cyclic version of Deligne's conjecture via cacti. Preprint 2004, arXiv:math.QA/0403340

[K3] R. M. Kaufmann, On several varieties of cacti and their relations. Algebr. Geom. Topol. 5 (2005), 237-300. Zbl 1083.55007 MR 2135554

[K4] R. M. Kaufmann, On spineless cacti, Deligne's conjecture and Connes-Kreimer's Hopf algebra. Topology 46 (2007), 39-88. Zbl 05123990 MR 2288726

[K5] R. M. Kaufmann, Moduli space actions on the Hochschild co-chains of a Frobenius algebra I: cell operads. J. Noncommut. Geom. 1 (2007), 333-384. Zbl 05238580 MR 2314100

[K6] R. M. Kaufmann, Dimension vs. genus: a surface realization of the little $k$-cubes and an $E_{\infty}$ operad. Preprint 2008, arXiv:0801.0532; The arc spectrum. In preparation.

[KLP] R. M. Kaufmann, M. Livernet, and R. C. Penner, Arc operads and arc algebras. Geom. Topol. 7 (2003), 511-568. Zbl 1034.18009 MR MR2026541

[KP] R. M. Kaufmann and R. C. Penner, Closed/open string diagrammatics. Nuclear Phys. B 748 (2006), 335-379. Zbl 05151248 MR MR2242677

[KSch] R. M. Kaufmann and R. Schwell, Associahedra, cyclohedra and a topological solution to the $A_{\infty}$-Deligne conjecture. Preprint 2007. arXiv:0710.3967

[Ko1] M. Kontsevich, Intersection theory on the moduli space of curves and the matrix Airy function. Comm. Math. Phys. 147 (1992), 1-23. Zbl 0756.35081 MR 1171758

[Ko2] M. Kontsevich, Formal (non)commutative symplectic geometry. In The Gel'fand Mathematical Seminars, 1990-1992, Birkhäuser, Boston 1993, 173-187. Zbl 0821.58018 MR 1247289

[Ko3] M. Kontsevich, Feynman diagrams and low-dimensional topology. In First European congress of mathematics (Paris, 1992), Vol. II, Progr. Math. 120, Birkhäuser, Basel 1994, 97-121. Zbl 0872.57001 MR 1341841

[Ko4] M. Kontsevich, Operads and motives in deformation quantization. Lett. Math. Phys. 48 (1999), 35-72. Zbl 0945.18008 MR 1718044

[KM] M. Kontsevich and Y. Manin, Gromov-Witten classes, quantum cohomology, and enumerative geometry. Comm. Math. Phys. 164 (1994), 525-562. Zbl 0853.14020 MR 1291244

[KS1] M. Kontsevich and Y. Soibelman, Deformations of algebras over operads and the Deligne conjecture. In Conférence Moshé Flato 1999 (Dijon), Vol. I, Math. Phys. Stud. 21, Kluwer Academic Publishers, Dordrecht 2000, 255-307. Zbl 0972.18005 MR 1805894 
[KS2] M. Kontsevich and Y. Soibelman, Notes on $A_{\infty}$-categories and non-commutative geometry. Manuscript.

[L] J-L. Loday, Cyclic homology. Second ed., Grundlehren Math. Wiss. 301. SpringerVerlag, Berlin 1998. Zbl 0885.18007 MR 1600246

[MSS] M. Markl, S. Shnider and J. Stasheff, Operads in algebra, topology and physics. Math Surveys Monogr. 96, Amer. Math. Soc., Providence, RI, 2002. Zbl 1017.18001 MR 1898414

[MS1] J. E. McClure and J. H. Smith, A solution of Deligne's Hochschild cohomology conjecture. In Recent progress in homotopy theory (Baltimore, MD, 2000), Contemp. Math. 293, Amer. Math. Soc., Providence, RI, 2002, 153-193. Zbl 1009.18009 MR 1890736

[MS2] J. E. McClure and J. H. Smith, Multivariable cochain operations and little $n$-cubes. J. Amer. Math. Soc. 16 (2003), 681-704. Zbl 1014.18005 MR 1969208

[MS3] J. E. McClure and J. H. Smith, Cosimplicial objects and little $n$-cubes. I. Amer. J. Math. 126 (2004), 1109-1153. Zbl 1064.55008 MR 2089084

[Me1] S. A. Merkulov, Frobenius $\infty$ invariants of homotopy Gerstenhaber algebras, I. Duke Math. J. 105 (2000), 411-461. Zbl 1021.53062 MR 1801768

[Me2] S. A. Merkulov, De Rham model for string topology. Internat. Math. Res. Notices 2004 (2004), 2955-2981. Zbl 1066.55008 MR 2099178

[P1] R. C. Penner, The decorated Teichmüller space of punctured surfaces. Commun. Math. Phys. 113 (1987), 299-339. Zbl 0642.32012 MR 0919235

[P2] R. C. Penner, Decorated Teichmüller theory of bordered surfaces. Comm. Anal. Geom. 12 (2004), 793-820. Zbl 1072.32008 MR 2104076

[St] K. Strebel, Quadratic differentials. Ergeb. Math. Grenzgeb. (3) 5, Springer-Verlag, Berlin 1984. Zbl 0547.30001 MR 0743423

[S1] D. Sullivan, Open and closed string field theory interpreted in classical algebraic topology. In Topology, geometry and quantum field theory (Oxford, 2002), London Math. Soc. Lecture Note Ser. 308, Cambridge University Press, Cambridge 2004, 344-357. Zbl 1088.81082 MR 2079379

[S2] D. Sullivan, Sigma models and string topology. In Graphs and patterns in mathematics and theoretical physics, Proc. Sympos. Pure Math. 73, Amer. Math. Soc., Providence, RI, 2005, 1-11. Zbl 1080.53085 MR 2131009

[T] D. E. Tamarkin, Another proof of M. Kontsevich formality theorem. Peprint 1998, arXiv:math/9803025; Formality of chain operad of little discs. Lett. Math. Phys. 66 (2003), 65-72. Zbl 1048.18007 MR 2064592

[TZ] T. Tradler and M. Zeinalian, On the cyclic Deligne conjecture. J. Pure Appl. Algebra 204 (2006), 280-299. Zbl 02242211 MR 2184812

[V1] A. A. Voronov, Homotopy Gerstenhaber algebras. In Conférence Moshé Flato 1999 (Dijon), Vol. II, Math. Phys. Stud. 22, Kluwer Academic Publishers, Dordrecht 2000, 307-331. Zbl 0974.16005 MR 1805923

[V2] A. A. Voronov, Notes on universal algebra. In Graphs and patterns in mathematics and theoretical physics, Proc. Sympos. Pure Math. 73, Amer. Math. Soc., Providence, RI, 2005, 81-103. Zbl 1083.18005 MR 2131012 
[W] C. Westerland, Equivariant operads, string topology, and Tate cohomology. Math. Ann. 340 (2008), 97-142. Zbl 05218952 MR 2349769

Received September 4, 2006; revised March 11, 2008

R. M. Kaufmann, Purdue University, West Lafayette, IN 47907, U.S.A.

E-mail: rkaufman@math.purdue.edu 